\title{
1 Cargo modulates the conformation of the nuclear pore in living cells
}

Authors: Joan Pulupa ${ }^{1}$, Harriet Prior ${ }^{1}$, Daniel S. Johnson², Sanford M. Simon ${ }^{1, *}$.

\section{Affiliations:}

${ }^{1}$ Laboratory of Cellular Biophysics, Rockefeller University, New York, NY 10065, USA.

2Department of Physics and Astronomy, Hofstra University, Hempstead, NY 11549, USA.

\section{Author List Footnotes:}

*Lead Contact: simon@mail.rockefeller.edu

\section{Abstract:}

11 While the static structure of the nuclear pore complex (NPC) continues to be refined

12 with cryo-EM and x-ray crystallography, the in vivo conformational dynamics of the NPC

13 remain under-explored. We developed sensors that report on the orientation of NPC

14 components by rigidly conjugating mEGFP to different NPC proteins. Our studies show

15 conformational changes to select domains of Nups within the inner ring (Nup54, Nup58,

16 Nup62) when transport through the NPC is perturbed and no conformational changes to

17 Nups elsewhere in the NPC. Our results suggest that select components of the NPC are

18 flexible and undergo conformational changes upon engaging with cargo.

\section{Introduction:}


Recent advances in structural biology have allowed the characterization of the static structures of large macromolecular complexes. However, describing the dynamics of proteins within these complexes, especially when the proteins are in their native cellular context, has proven challenging. In this paper, we establish a technique to visualize the orientations of domains of proteins in vivo and we apply this technique to study the conformational dynamics in the nuclear pore complex (NPC).

The NPC is a cylindrical channel composed of proteins that form an eight-spoked core with an axis perpendicular to the nuclear envelope (Alber et al., 2007; Kim et al., 2018; Kosinski et al., 2016; Mosalaganti et al., 2018). NPCs are composed of $~ 30$ distinct nucleoporins (Nups) represented in 8, 16, or 32-fold copy number. An individual

11 yeast NPC is composed of $\sim 500$ total Nups, whereas a vertebrate NPC has $\sim 1,000$ total

12 Nups (Hoelz et al., 2011). The yeast NPC is 66 MDa (Rout \& Blobel, 1993), while the

13 mammalian NPC is $\sim 109$ MDa (Reichelt et al., 1990). While the organizing principles of

14 the NPC are shared across eukaryotes, there are notable variations even between

15 human cell lines. Differential expression levels (D'Angelo et al., 2012; Lupu et al., 2008) and stoichiometries (Ori et al., 2013) of Nups are observed between different cell types.

17 Furthermore, within the mammalian NPC, different Nups have been shown to have

18 different residence times, which has led to the suggestion that those with shorter

19 residence times may serve adaptor or regulatory functions (Rabut et al., 2004). The

20 additional mass of the mammalian NPC exists in the absence of substantial changes to

21 the size of the central channel, raising the possibility that it is potentially subject to

22 additional regulation. 
In the adherent cell lines used in this study, the nuclei tend to be flattened ovoids and the NPCs on the basal surface share a common orientation with their central axis perpendicular to the coverslip. Within each NPC, each component is in 8-fold symmetry. Therefore, the NPC is a compelling test structure to assay conformational changes in vivo. Using polarized-total internal reflection microscopy (pol-TIR), we monitored the orientation of mEGFP-based sensors incorporated into different domains of individual response to both transport state and to specific transport factors. To monitor conformational changes in the scaffold of the NPC, we built orientational sensors by rigidly attaching mEGFP to different Nup domains. We conjugated the alpha helix at the

13 amino terminus of mEGFP to the carboxyl-terminus of a Nup domain. Thus, the orientation of mEGFP is fixed to that of the Nup. The orientation of mEGFP can be monitored because the excitation dipole is fixed within the molecule. The strength of excitation light and the excitation dipole. To monitor the orientation of the mEGFP, we excited the fluorophore sequentially with light polarized in two orthogonal directions. This technique has been once by our group to look at dynamics of assembly of HIV-1 in vivo (Johnson et al., 2018). Previously, to measure the organization of various Nups with respect to the NPC we used fluorescence anisotropy. We determined the organization of the Y-shaped complex with respect to the NPC (Kampmann et al., 2011) and characterized the orientations and rigidity of the FG-Nup domains (Atkinson et al., 
1 2013; Mattheyses et al., 2010). The anisotropy approach used polarized light to excite

2 many dozens of NPCs and then measure the emission parallel and perpendicular to the

3 excitation.

$4 \quad$ In this work, we determine the orientation of proteins within individual NPCs by

5 rapidly switching the excitation between two orthogonal polarizations. We created an

6 evanescent field with total internal reflection (TIR) that allows us both to control

7 polarization and restrict excitation to the basal surface of the cell. Because the basal

8 nuclear envelope is parallel to our coverslip, the NPCs embedded in the nuclear

9 envelope are oriented relative to the optical axis of our microscope. Therefore, it is

10 possible, for the first time, for us to monitor the orientation of Nups within individual

11 NPCs in living cells. Our results show a structural rearrangement of the structural core

12 of the NPC, in particular of the inner ring Nups, in response to manipulations of

13 transport through the NPC. 
1 Results:

2 The Orientation of Nup-mEGFP Fusion Proteins Can be Monitored with Polarized-

3 Total Internal Reflection Fluorescence Microscopy (pol-TIRFM)

We alternated the polarization of the excitation field between $\widehat{\mathbf{p}}$-polarized light

(perpendicular to the coverslip and parallel to the nucleo-cytoplasmic axis of each NPC)

6 and $\hat{\mathbf{s}}$-polarized light (parallel to the coverslip and perpendicular to the nucleo-

7 cytoplasmic axis of each NPC). We then measured the light emitted from each Nup

8 orientational sensor in response to the two orthogonal polarizations of TIRF illumination

9 (Figure 1A).

10 


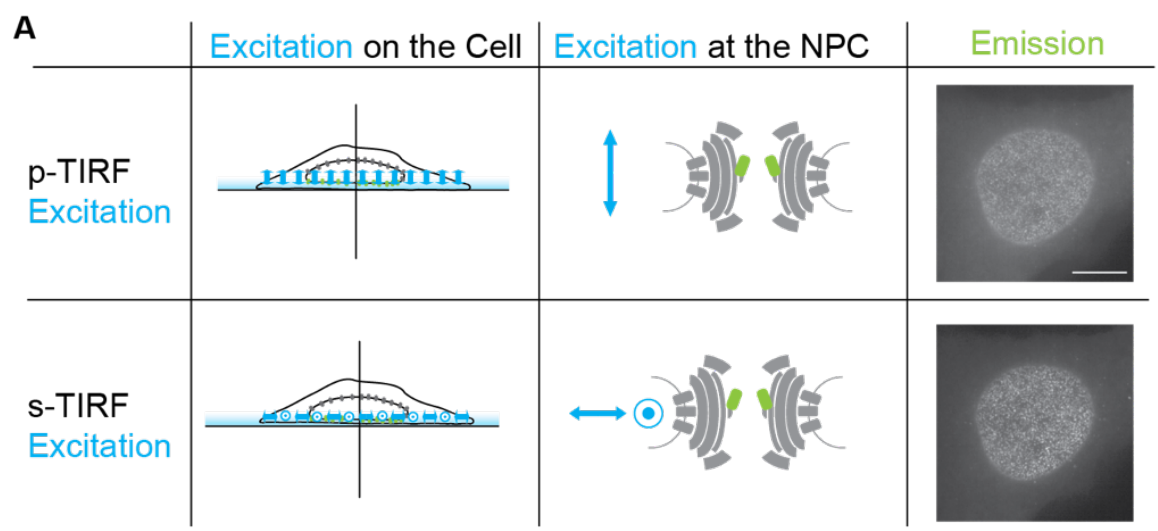

B

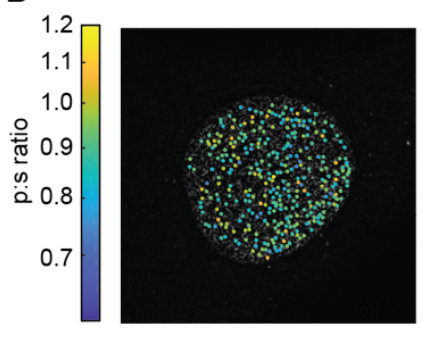

C

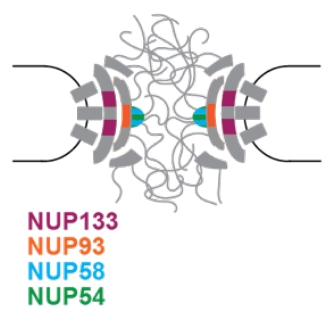

D

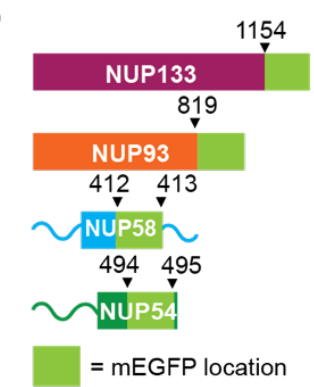

\begin{tabular}{c|c|l}
\multirow{2}{*}{$E$} & $\begin{array}{l}\text { Net } \\
\text { Fusion } \\
\text { Size }\end{array}$ & $\begin{array}{l}\text { Sequence } \\
\text { Nup54LinkerGFP }\end{array}$ \\
\hline \multirow{2}{*}{ Rigid } & OAA & DIKLVAEAAAEELFT \\
\cline { 2 - 3 } Linkers & $1 \mathrm{AA}$ & DIKLVAEAAAKEELFT \\
\cline { 2 - 3 } & $2 \mathrm{AA}$ & DIKLVAEAAAKEEELFT \\
\hline \multirow{2}{*}{$\begin{array}{c}\text { Flexible } \\
\text { Linkers }\end{array}$} & OAA & DIKLVGGGGSEELET \\
\cline { 2 - 3 } & $1 \mathrm{AA}$ & DIKLVGGGGSSEELFT \\
\cline { 2 - 3 } & 2AA & DIKLVGGGGSSGEELFT \\
\hline
\end{tabular}
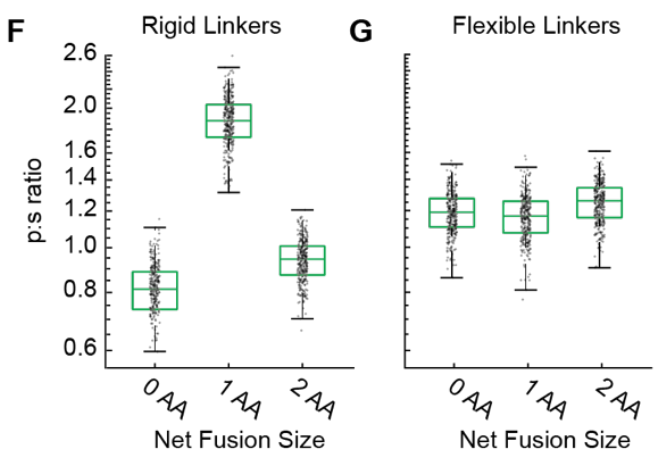
pol-TIRF.

Figure 1: The orientation of Nup-mEGFP fusion proteins can be measured in individual NPCs with

A) Using pol-TIRF, the bottom of the nucleus is illuminated and Nup-mEGFP fusion proteins are excited with $\widehat{\mathbf{p}}$-polarized or $\widehat{\mathbf{s}}$-polarized light. $\widehat{\mathbf{p}}$-excitation is parallel and $\widehat{\mathbf{s}}$-excitation is perpendicular to the nucleocytoplasmic axis of the NPC. The emission from Nup54-mEGFP fusion proteins in HeLa cells in response to each excitation are shown in the right column. (Scale bar $=10 \mu \mathrm{m})$. B) p:s ratios, a measurement of orientation of mEGFP, are calculated for each NPC and represented with a color scale. C) Schematic of the NPC indicating the Nups studied. D) Schematic of the mEGFP placement within the Nups. E) Nup54-mEGFP constructs with flexible or rigid linkers in between the Nup and the mEGFP. With 
bioRxiv preprint doi: https://doi.org/10.1101/2020.07.27.223081; this version posted July 28,2020 . The copyright holder for this preprint (which

was not certified by peer review) is the author/funder, who has granted bioRxiv a license to display the preprint in perpetuity. It is made available under aCC-BY 4.0 International license.

1 each additional amino acid in a rigid alpha helix, the mEGFP rotates $103^{\circ}$ relative to the Nup, which does

2 not happen with a flexible linker. F) The p:s ratios of Nup54-mEGFP ${ }^{494}$ fusion proteins with a rigid linker

3 shift with the addition of each amino acid. G) The p:s ratios of Nup54-mEGFP ${ }^{494}$ fusion proteins with a

4 flexible linker do not shift upon amino acid additions. ( $n=300$ NPCs, 10 cells, boxes indicate quartiles,

5 center bars indicate medians).

6 Figure 1-figure supplement 1. The Orientation of Nup-mEGFP Fusion Proteins can be Monitored with 7 pol-TIRFM. 
We probed for conformational changes in the NPC with orientational sensors in

Nup133, Nup93, Nup54, and Nup58. Nup133 is a member of the Y-shaped complex, which forms two reticulated rings at both the nuclear and cytoplasmic faces of the NPC (Bui et al., 2013). Nup93 is a member of the Nup93 complex (along with Nup205, Nup188, Nup155, and Nup53) and Nup54 and Nup58 are members of the Nup62 complex (along with Nup62). Both the Nup93 and Nup62 complexes localize to the inner ring (Vollmer \& Antonin, 2014), between the two reticulated rings of $Y$-shaped complexes. The Nup93 complex is embedded within the NPC scaffold, and the Nup62 complex sits adjacent, closer to the lumen of the nuclear pore (Kosinski et al., 2016). Therefore, our orientational sensors were localized to three different structural positions within the NPC (Figure 1C).

Plasmids encoding putative orientational sensors were engineered to encode each Nup fused via an alpha helix to mEGFP. We removed the first 4 amino acids of the mEGFP because they are not resolved in the crystal structure, suggesting that they are not rigid. We transfected these plasmids into HeLa cells (Figure 1A, Figure 1—figure supplement 1). We wrote an algorithm (see Methods) to detect individual NPCs and extract light emitted in response to perpendicular $(\widehat{\mathbf{p}})$ excitation and parallel $(\widehat{\mathbf{s}})$ excitation to calculate what we refer to as the p:s ratio (Figure 1B, Figure 1-figure supplement 1). If the mEGFP is rigidly conjugated to a Nup that is properly incorporated into the NPC, then changes of the p:s ratio represent changes in orientation of the domain of the Nup to which the mEGFP is conjugated.

If the mEGFP is held rigidly relative to a Nup, then changing the length of the alpha helical linker by a single amino acid should rotate the mEGFP $\sim 103^{\circ}$ around the 
1 axis of the alpha helix. Therefore, if our reporter is a proxy for the orientation of the Nup,

2 the p:s ratio should shift with the number of amino acids in the linker. This hypothesis

3 was tested by inserting rigid or flexible linkers of different lengths between mEGFP and

4 the terminal structured alpha helix of Nup54 (Figure 1E), which is defined as residues

5 456-494 (Solmaz et al., 2011). The constructs were transiently transfected into HeLa

6 cells, and the p:s ratios were measured in living cells 24-48 hours post transfection. The

7 p:s ratio shifted with different lengths of the rigid linkers, confirming that the dipole of the

$8 \quad$ mEGFP is a proxy for the orientation of the Nup54 (Figure 1F). No difference in p:s ratio

9 was observed with varying lengths of the flexible linkers (Figure 1G). Thus, we

10 developed a criterion whereby orientational sensors are considered functional if the p:s

11 ratio shifts upon the addition of a single amino acid.

Using this criterion, we confirmed that we have orientational sensors with

13 mEGFP conjugated to Nup133, Nup93, Nup58, and Nup54 (Figure 2A-D). We saw

14 distinct shifts in the p:s ratio when constructs differed by a single amino acid. An

15 exception was when mEGFP was placed at the carboxyl terminus of Nup54. This

16 domain is currently unresolved in any crystal structure, and the p:s ratio did not change

17 upon rotating the linker alpha helix (Figure 2E). This indicates that this domain of Nup54

18 is not held rigidly, consistent with the inability to crystalize this domain of the protein and

19 suggests the carboxyl terminus is a flexible domain. For any specific Nup-mEGFP,

20 variations in the p:s ratio in different NPC were not detected with respect to position

21 along the basal surface of the nucleus (Figure 1-figure supplement 1 ), indicating a

22 shared orientation for these NPCs. 
A Nup133-mEGFP और 数

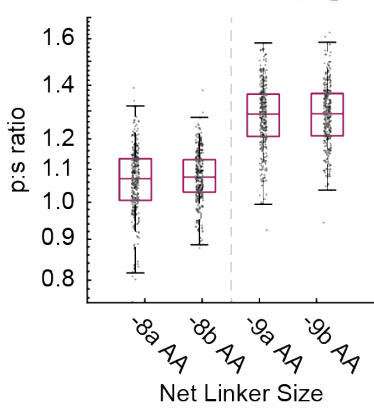

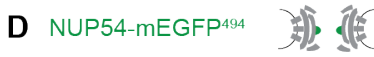

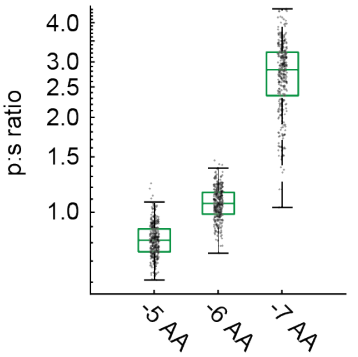

Net Linker Size

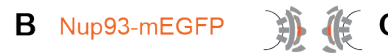

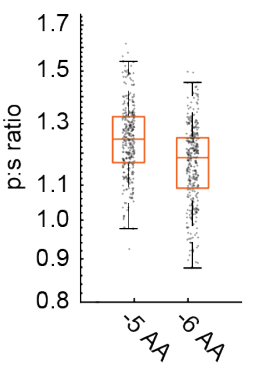

Net Linker Size

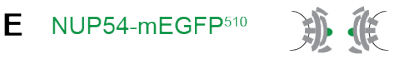

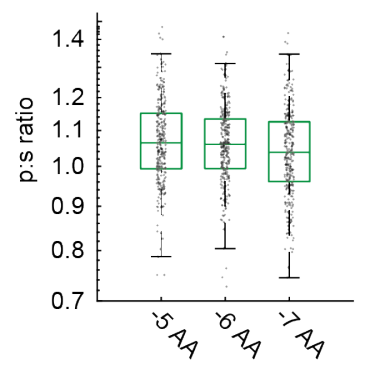

Net Linker Size

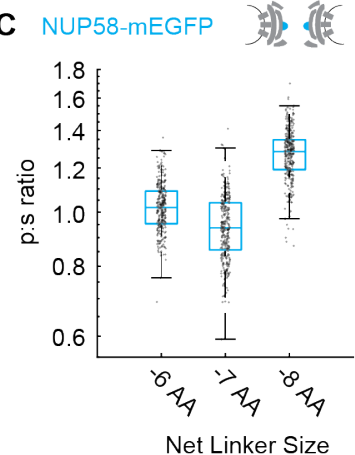

Net Linker Size

Figure 2: Varying the length of the linker between the Nup and the mEGFP by single amino acids to test validity of the orientational sensors.

A) Nup133-mEGFP, with linkers of different lengths at its carboxyl terminus, conjugated to mEGFP. A change in the linker length by a single amino acid changes the p:s ratio. Pairs of different rigid alpha helical linkers of the same length generate indistinguishable p:s ratios. B) Nup93-mEGFP with different linker lengths to mEGFP at the carboxyl terminus. One deleted amino acid shifts the p:s ratio. C) Nup-58 with the mEGFP at the carboxyl terminus of the coiled-coiled domain. Each subsequent deletion of a single amino acid alters the p:s ratio. D) Nup54-mEGFP ${ }^{494}$ with the mEGFP at the carboxyl terminus of

11 the coiled-coiled domain (Amino Acid: 494). Each subsequent deletion of a single amino acid changes the

12 p:s ratio. E) Nup54-mEGFP 510 with the mEGFP at the carboxyl terminus of the protein (Amino Acid: 510).

13 Each subsequent deletion of a single amino acid does not alter the p:s ratio. ( $n=300$ NPCs, 10 cells,

14 boxes indicate quartiles, center bars indicate medians). 


\section{The Orientations of Inner Ring Nups Change After Starvation}

In yeast, starvation inhibits the import of cargo with nuclear localization sequences, or NLS-tagged cargo (Stochaj et al., 2000). We tested the effects of starvation on the orientation of Nups. Cells were starved for 24 hours in Hank's Balanced Salt Solution (HBSS) and we confirmed the rates of intracellular transport 2015) (Figure 3-figure supplement 1). and Nup93 (Figure 3A-B, Figure 3-figure supplement 2A-D). These results are

11 consistent with the NPC retaining its orientation relative to the coverslip. The consistency across the basal surface of the nucleus shows the nuclear envelope is not

13 distorted post-starvation. (Nup54-mEGFP ${ }^{494}$, or Nup54 ${ }^{494}$ ) exhibited significant shifts post-starvation (Figure 3 CD, Figure 3-figure supplement 2E-H). These orientational shifts in select alpha helices of Nup54 and Nup58 reflected a reorganization of these inner ring Nups relative to the NPC. No shift was observed in the previously described reporter at amino acid 510 in Nup54 (Nup54-mEGFP510, or Nup54 ${ }^{510}$ ), the carboxyl terminus (Figure 3E). These results suggest a change of orientation of select alpha-helical domains of Nup58 and

21 Nup54 in the absence of a change in the orientation of the rest of the NPC. 


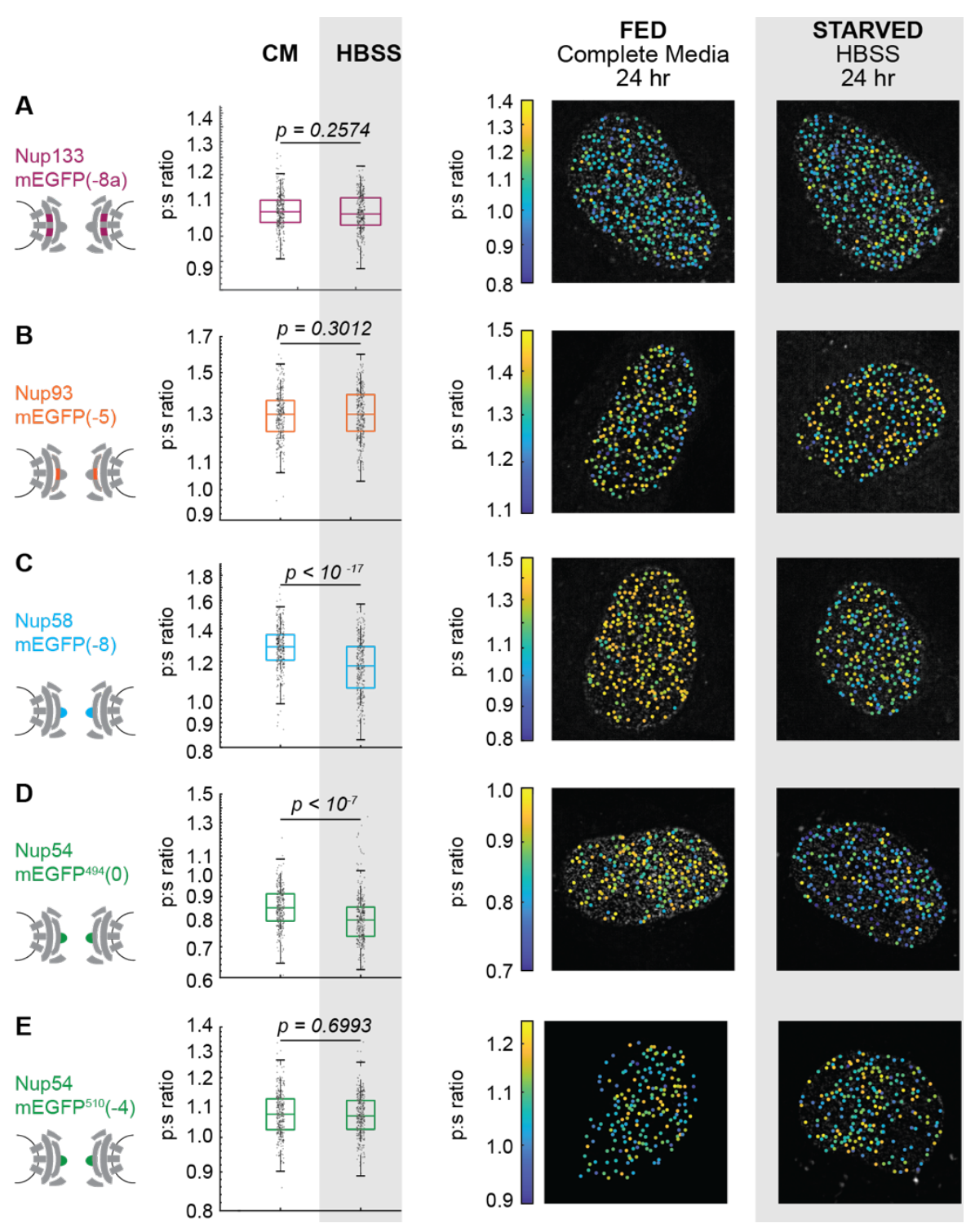

Figure 3: The Inner Ring Nups, Nup54 and Nup58, are reorganized with respect to the NPC after starvation.

4 Cells were maintained in complete media (CM) or starved for 24 hours in HBSS prior to imaging. The p:s

5 ratios and representative images are presented for: (A) Nup133-mEGFP(-8a), (B) Nup93-mEGFP(-7), (C)

6 Nup58-mEGFP(-8), (D) Nup54-mEGFP ${ }^{494}(0)$, and (E) Nup54-mEGFP $510(-4)$. The orientation changed for

7 Nup58-mEGFP and Nup54-mEGFP ${ }^{494}$. For additional linker lengths, see Figure 3-figure supplement 2.

8 HeLa cells were imaged 48 hours post transfection. ( $n=300$ NPCs, 10 cells, boxes indicate quartiles,

9 center bars indicate medians, student $t$-test). 
bioRxiv preprint doi: https://doi.org/10.1101/2020.07.27.223081; this version posted July 28, 2020. The copyright holder for this preprint (which was not certified by peer review) is the author/funder, who has granted bioRxiv a license to display the preprint in perpetuity. It is made available under aCC-BY 4.0 International license.

1 Figure 3-figure supplement 1. Nuclear Transport is Attenuated Post-Starvation.

2 Figure 3-figure supplement 2. The Inner Ring Nups, Nup54 and Nup58, are reorganized with respect

3 to the NPC after starvation.

4 


\section{Nup-mEGFP Orientational Reporters are Functional}

To ensure that the Nup-mEGFP fusion proteins are functional and to improve the signal to noise, we used CRISPR/Cas9 to create cell lines in which both endogenous copies of either Nup133 or Nup54 are replaced with their fluorescent orientational sensor equivalent.

We confirmed that these cell lines are homozygous (Figure 4-figure supplement 1) and observed no changes in cell growth or morphology (Figure 4 A-B). These cell lines also shared the property that single amino acid alterations in the linker length shifted the p:s ratio (Figure 4 C-D). When starved, the CRISPR cell lines mimicked the results of the transiently transfected cell lines (Figure 4 E-F, Figure 4-figure

11 supplement 2); the orientation of Nup54 ${ }^{494}$ exhibited a significant change but the

12 orientation of the Nup133 did not shift after starvation. Just as in the transient

13 transfections, Nup54 was reorganized when transport was reduced.

\section{The Orientation of Inner Ring Nup54 Changes After Blocking Nuclear Export} orientation of domains of Nup54, we used three additional approaches: permeabilization of the plasma membrane with digitonin, treatment with leptomycin $B$, and expression of membrane intact, resulting in the loss of many cytoplasmic components and the

21 cessation of nuclear-cytoplasmic trafficking (Adam et al., 1992). After digitonin

22 permeabilization, we monitored cells to ensure that the nuclear envelope remained 
1 intact (Figure 5-figure supplement 1A) and we confirmed that the cells were capable of translocating specific NLS-tagged cargos (Figure 5-figure supplement 1D-E). When we permeabilize cells and incubate in a buffer containing no transport factors or cargo, we observed a distinct shift in the orientation of Nup54 ${ }^{494}$ in the same direction as starvation but no change in the orientation of Nup133 (Figure $4 \mathrm{G}-\mathrm{H}$ ). evolutionarily conserved export receptor (Kudo et al., 1999). We treated our cells with $25 \mathrm{nM}$ leptomycin for 15 hours and observed an orientational shift of Nup54 ${ }^{494}$ but no change to the orientation of Nup133 (Figure 4 l-J).

To disrupt both import and export, we expressed a dominant negative Ran. Ran

11 is a GTPase that mediates nuclear transport (Moore \& Blobel, 1993). Ran undergoes

12 GTP hydrolysis in the cytosol that causes the Ran-Kap1- $\beta$ export complex to dissociate

13 from the NPCs, thereby replenishing Kap1- $\beta$ in the cytosol. The Ran-Q69L mutant

14 cannot perform GTP-hydrolysis, thereby blocking Ran-dependent import and export

15 (Bischoff et al., 1994). not capable of nuclear-cytoplasmic trafficking by using a light induced nuclear shuttle

We confirmed that cells transiently expressing BFP-RanQ69L for 24 hours were (Figure $4 \mathrm{M}-\mathrm{N}$ ). Nup54 ${ }^{494}$ was rearranged once again with respect to the NPC but the Nup133 did not change orientation after cells were transfected with a DN-Ran (Figure 4 $\mathrm{K}-\mathrm{L})$.

These results suggest that attenuating the transport of NLS-driven cargo shifts the orientation of select alpha helical domains of inner ring Nups. The direction of the 
bioRxiv preprint doi: https://doi.org/10.1101/2020.07.27.223081; this version posted July 28, 2020. The copyright holder for this preprint (which was not certified by peer review) is the author/funder, who has granted bioRxiv a license to display the preprint in perpetuity. It is made available under aCC-BY 4.0 International license.

1 p:s ratio shift of Nup54-mEGFP ${ }^{494}$ is consistent among all mechanisms of reducing

2 cargo flux.

3

4 


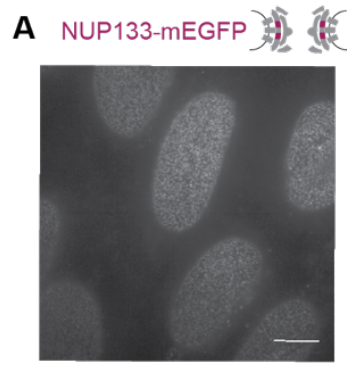

C

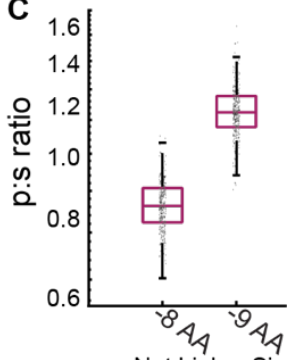

B NUP54-mEGFP494
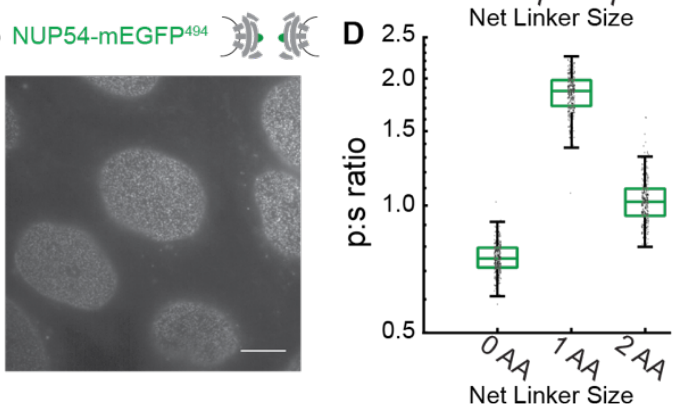

E CM HBSS
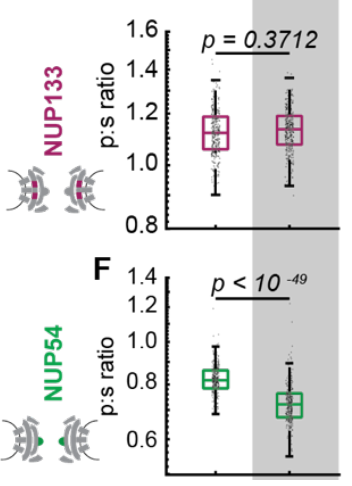

G

CM PERM I

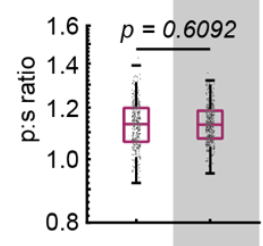

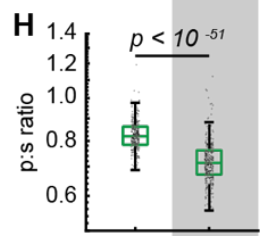
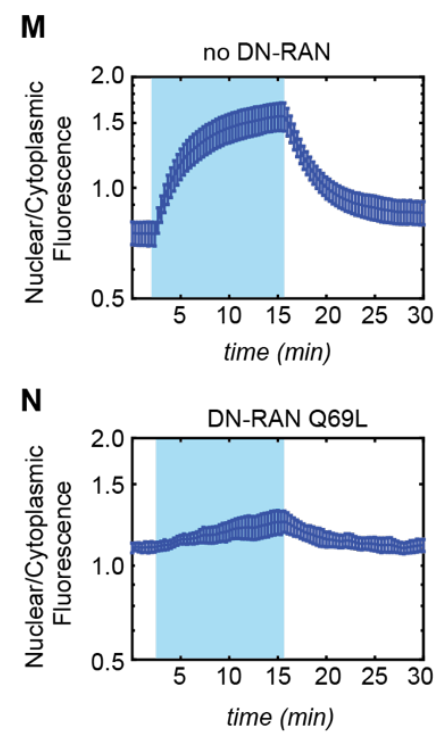

CM LEP B $\mathrm{K}$
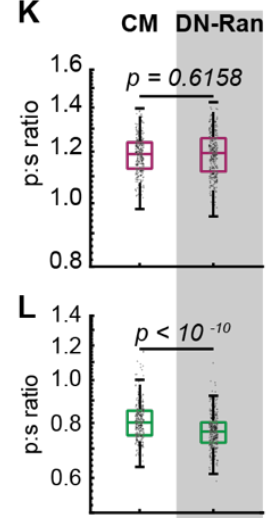

Figure 4. Conformational dynamics of the Inner Ring of the NPC revealed by perturbations of cargo state in CRISPR cell lines.

A-B) No morphological distortions are detected in cell lines endogenously expressing orientational sensors. C) Nup133-mEGFP cell lines with the mEGFP at the carboxyl terminus of the protein with different linker lengths. One deleted amino acid shifts the p:s ratio. D) Nup54-mEGFP ${ }^{494}$ cell lines with the mEGFP at the carboxyl end of the coiled-coiled domain of the protein with constructs of different linker lengths. One deleted amino acid shifts the p:s ratio. E-F) Orientational sensor cell lines were maintained in CM or starved for 24 hours in HBSS. G-H) CRISPR cell lines mock permeabilized or digitonin permeabilized prior to imaging. I-J) CRISPR cell lines mock treated or treated with leptomycin B prior to imaging. K-L) CRISPR cell lines with or without transient expression of dominant negative Ran. ( $n=300$ 
bioRxiv preprint doi: https://doi.org/10.1101/2020.07.27.223081; this version posted July 28,2020 . The copyright holder for this preprint (which

was not certified by peer review) is the author/funder, who has granted bioRxiv a license to display the preprint in perpetuity. It is made available under aCC-BY 4.0 International license.

$1 \quad$ NPCs, 10 cells, boxes indicate quartiles, center bars indicate medians, student $t$-test). M-N) The average

2 change in the ratio of nuclear/cytoplasmic fluorescence in HeLa cells with and without dominant negative

3 Ran transiently expressed ( $n=6$, mean reported \pm SEM with error bars). The shaded region represents the

4 time of blue light LANS activation.

Figure 4-figure supplement 1. Validation of CRISPR Cell Lines.

7 Figure 4-figure supplement 2. The Inner Ring Nup Nup54 is reorganized with respect to the NPC after 8 starvation.

9 


\section{Altering Karyopherin Content at the NPC Changes the Conformation of Nup54}

After digitonin permeabilization and removal of cytosol, a pool of endogenous karyopherins (kaps) remain associated with the NPCs for a period of hours (Kapinos et al., 2017). These kaps can be dissociated from the NPC by introducing Ran-GTP to the nuclear periphery (Figure 5A, Figure 5-figure supplement 1F-H). Then, the kap

6 population can be restored by adding exogenous kaps, which we have purified and have determined to be capable of transporting NLS-tagged cargo (Figure 5-figure supplement 1D-E). We have chosen to reintroduce Kap1- $\beta$ and Kap1- $\alpha$. The Kap- $\beta$ (or importin- $\beta$ ) family of kaps encompasses over 20 proteins in mammalian cells. We reintroduced Kap1- $\beta$ a 97-kDa import receptor that regulates the canonical import pathway (Kimura et al., 2017). Kap1- $\beta$ interacts with Kap1- $\alpha$, a 58-kDa adaptor that acts to import classic NLS-tagged cargos (Pumroy \& Cingolani, 2015). and restoration of kaps reversed this shift (Figure $5 \mathrm{~F}-\mathrm{I}$ ). Upon addition of kaps alone,

15 the orientation of Nup54 ${ }^{494}$ shifted back towards its position prior to permeabilization. Although the orientation shift does not totally shift the orientation back towards the initial

17 orientation, we are only supplying two of the many species of kaps present in the NPC

18 in a living cell and no NLS-tagged cargo. In contrast, dissociation or restoration of kaps at the NPC did not produce changes in the orientation of Nup133 (Figure 5 B-E). 

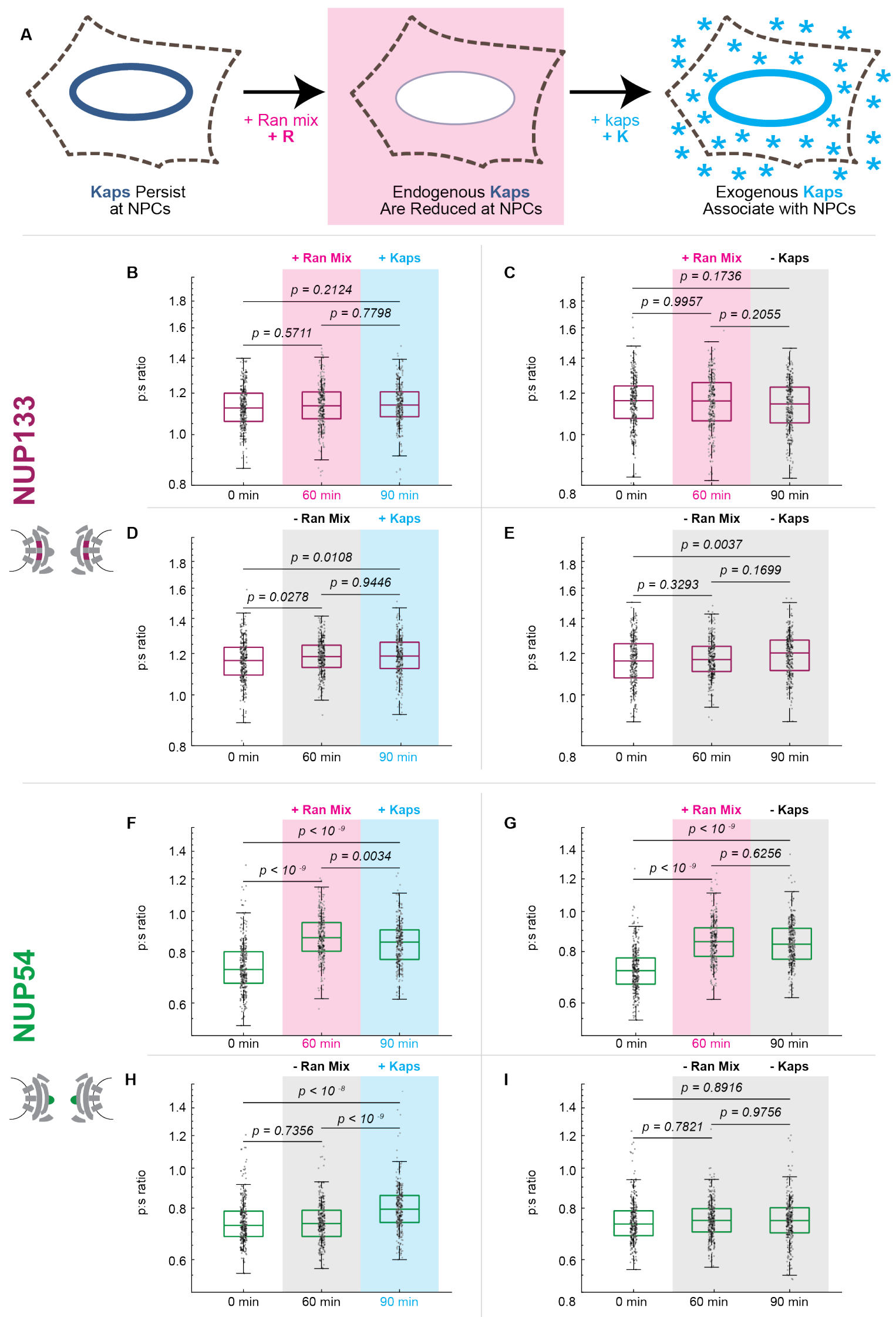
1 Figure 5: Karyopherin content at the nuclear periphery induces conformational changes in Nup54-

2 mEGFP ${ }^{494}$ but not Nup133-mEGFP.

3 A) Digitonin permeabilization allows the introduction of transport factors to nuclear periphery. B-E)

4 Nup133-mEGFP does not experience a shift in orientation after removal of endogenous kaps or addition

5 of exogenous kaps. F-I) Nup54-mEGFP ${ }^{494}$ changes after removal of endogenous kaps or introduction of

6 exogenous kaps. Pink boxes indicate the addition of Ran mix, blue boxes indicate the addition of kaps,

7 and gray boxes indicate a buffer change with no additional transport factors. ( $n=300$ NPCs, 30 cells,

8 boxes indicate quartiles, center bars indicate medians, one-way ANOVA with post-hoc Tukey test).

9

10 Figure 5-figure supplement 1. Nuclear transport factors are be introduced to nuclear periphery using

11 digitonin permeabilization of the plasma membrane. 


\section{The Arrangement of Inner Ring Nup62 Changes After Starvation}

To test whether the orientational shifts in Nup54 ${ }^{494}$ were coincident with a spatial reorganization of inner ring Nups, we measured the proximity of Nup62s to each other by Förster resonance energy transfer (FRET). We engineered a homozygous cell line where Nup62 was replaced with a copy of Nup62 with FRET sensors (mEGFP 290 and

6 mCherry $^{321}$ ) on opposite sides of an alpha helical domain of Nup62 (Figure 6). This

7 alpha helix lies between the coiled-coiled anchor region and a flexible FG-repeat region

8 of Nup62 and is an elongated region that has been predicted to interact with a

9 structured region of Nup54 (Sharma et al., 2015).We measured FRET with acceptor

10 photobleaching and quantified FRET efficiency. The FRET efficiency of these sensors

11 experienced a shift, consistent with inner ring Nups experiencing a conformational shift

12 post-starvation (Figure 6). 
Nup62 FRET Probes

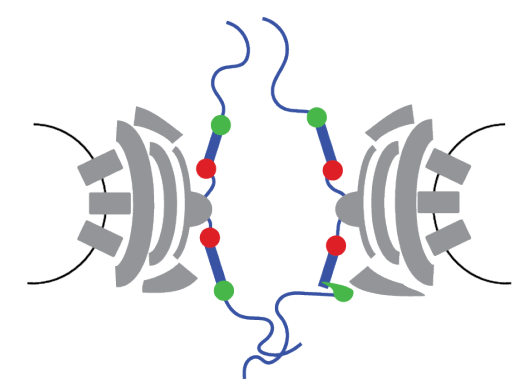

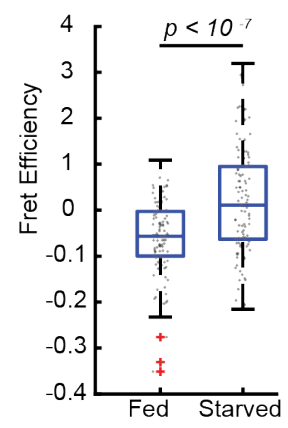

2 Figure 6. FRET between Nup62 'finger' domains increases after starvation.

3 Under starvation conditions, FRET increased between Nup62 'finger' domains. A) Schematic of Nup62

4 FRET probe labelling scheme. B) FRET efficiency for HeLa cells were imaged 48 hours post transfection.

5 Cells were kept in CM or starved for 24 hours in HBSS ( $n=100$ NPCs, 10 cells, boxes indicate quartiles,

6 bars indicate medians).

7 
Discussion:

A number of recent studies have reported variability in the organization and diameter of NPCs. Super resolution imaging has been used to visualize NPCs at different developmental stages of $X$. laevis oocytes, demonstrating that the organization and diameter of the NPCs changes over time (Selles et al., 2017). By cryo-ET using sub-tomogram averaging in either transport competent or transport inhibited cells, two distinct structural states are observed with differences in the central transporter, suggesting that this region might undergo conformational changes upon engagement of cargo (Eibauer et al., 2015; Zwerger et al., 2016). In HeLa cells, NPCs from the same cell were observed with cryo-ET to be more similar in inner diameter than those from other cells, suggesting that diameter might change as a result of a cell's specific physiological state (Mahamid et al., 2016). Structural studies based on two discrete crystal states of short peptides of three inner channel ring Nups, Nup58, Nup54, and Nup62, have led to the proposal that the structured regions of these inner ring Nups cycle between a dilated ring of $40-50 \mathrm{~nm}$ in diameter and a constricted ring of $20 \mathrm{~nm}$ in diameter. The Ring Cycle hypothesis suggests that this ring undergoes conformational changes and directly regulates cargo import and export (Melcak et al., 2007; Sharma et al., 2015; Solmaz et al., 2013; Solmaz et al., 2011). It has also been proposed that the evolution of the NPC into a complex sized over $\sim 109$ MDa was in part driven by the need to cushion the huge diameter changes of the central transport channel by a large and deformable surrounding protein matrix (Hoelz et al., 2011). 
The work presented here is the first time that altered conformations of the NPC have been shown in living cells. Furthermore, these experiments have been done at the level of single active NPCs in which altered conformations are correlated to the loading of kaps. Our results are consistent with orientational changes to the inner ring of the NPC in response to changes in transport state, similar to the orientational changes

6 present in the Ring Cycle. Although we cannot yet calculate the magnitude of the angle changes of these alpha helices, we can say with confidence that these alpha helices are experiencing a rearrangement with respect to the NPC after they are confronted with perturbations in transport. And that these orientational shifts are confined to the inner ring of the NPC, the most constricted portion of the NPC: Similar changes are not detected when probes are placed in other locations. These conformational changes may be coincident with cargo translocation or may be an indication that the scaffold of

13 the NPC serves as a dynamic gate that can regulate nuclear trafficking. The NPC structure as a whole may be modulated by cargo load and identity. Compositional and research in the field. on our previous work (Atkinson et al., 2013; Kampmann et al., 2011; Mattheyses et al., complex, and their effects on transport and regulation remain exciting future avenues of

The imaging technique presented here allows for the monitoring of Nup dynamics within individual NPCs simultaneously in a single living cell. This technique builds upon 2010) to provide the first tools to directly observe the conformations and orientational dynamics of Nups in living cells. By expanding this technique into pol-TIRF, we have developed the first tools to directly observe the orientational dynamics of Nups within a 
1 single NPC in living cells and to track these dynamics over time with different cargo

2 conditions.

This light microscopy technique can be used to probe the orientations of different

4 domains within an individual protein either on its own or as part of a macromolecular

5 complex. By allowing one to not only localize a protein, but to also monitor the

6 orientations of different domains within a protein, this technique can provide insights into

7 various molecular mechanisms. Polarization microscopy can be used to monitor the

8 dynamics and organization of domains of other macromolecular complexes in vivo and

9 in vitro, including the ribosome, proteasome, centriole, and cilia, while allowing these

10 complexes to dynamically respond to their environments and perform work. 


\section{Materials and Methods:}

2 Cell Lines and Growth derived Nup-mEGFP cell lines (this paper) were cultured in Dulbecco's Modified Eagle's Medium (DMEM, Gibco, Waltham, MA), supplemented with l-glutamine and sodium pyruvate (from here-on referred to as DMEM) and 10\% (vol/vol) fetal bovine serum (FBS, Sigma, St. Louis, MO) in humidified incubators at $37 \mathrm{C}$ and in a $5 \% \mathrm{pCO}_{2}$ atmosphere, using standard sterile techniques. HeLa cells were recently acquired from ATCC and Hap1 cell lines were acquired from Horizon technologies. Cells were negative for mycoplasma.

11 For starvation experiments, cells were imaged, then washed $3 x$ with PBS and

12 placed in 1x Hank's Balanced Salt Solution with calcium and magnesium (HBSS, Gibco)

13 for $24 \mathrm{~h}$ and imaged. For leptomycin B experiments, cells were either treated with $25 \mathrm{nM}$

14 leptomycin B (Sigma) or vector (methanol) for $15 \mathrm{~h}$, at which point both were imaged.

15 Imaging Conditions dishes were uncoated, but for Hap1 cells (all CRISPR cell lines) the dishes were coated

18 with fibronectin (Gibco). During imaging, the media was replaced with cell imaging media [HBSS (Sigma), 10 mM HEPES, pH7.4], supplemented with 10\% FBS (vol/vol,

20 Sigma).

\section{Microscopy: General}

Cells were imaged on a custom-built microscope, based on an inverted IX-81 frame

23 (Olympus Life Sciences, Tokyo, Japan) and equipped with a custom built through-the- 
1 objective (100X UAPON 1.49 NA, Olympus and 100x UAPON 1.51 NA, Olympus Life Sciences) polarized TIRFM illuminator equipped with a 405-nm laser (100 mW LuxX diode laser, Omicron, Rodgau-Dudenhofen, Germany), a 488-nm laser (100 mW LuxX diode laser, Omicron), a 594-nm laser (100 mW diode-pumped solid-state laser, Cobolt AB, Stockholm, Sweden), and a 647-nm laser (100 mW LuxX diode laser, Omicron) (Johnson et al., 2014). For live-cell and permeabilized cell experiments, the temperature was maintained at $37 \mathrm{C}$ throughout imaging using custom-built housing. galvanometers (Nutfield Technology, Cranberry Township, PA). An electro-optic modifier (EOM, Conoptics, Danbury, CT) and a quarter-wave-plate (Thorlabs, Newton, $\mathrm{NJ}$ ) before the galvanometers controlled the polarization of the $488 \mathrm{~nm}$ laser. The galvanometers, EOM, camera shutter, and 488 laser shutters were all driven by a multifunctional data acquisition board (PCle-6323, 577, National Instruments, Austin, TX) and controlled from custom written software in LabView (National Instruments) (Johnson et al., 2014). All emission light was collected after it was passed through a multiband polychroic (zt405/488/594/647rpc 2mm substrate, Chroma, Bellows Falls, VT) to isolate the excitation light from the emitted light. Images were collected on a CMOS camera (Flash-4.0, Hamamatsu Photonics, Middlesex, NJ) connected with Hamamatsu Camera Link interface to a workstation (Precision Model T7500, Dell, Austin, TX) running image acquisition software (Metamorph, Molecular Devices, San Jose, CA) (Johnson et al., 2014). Microscopy: pol-TIRF of mEGFP Nups 
A sequence of 20 images was taken with alternating $p$ - and s-excitation in TIR.

Each individual $\mathrm{p}$ or s image had an exposure time of $5 \mathrm{~ms}$ (laser power: $100 \mathrm{~mW}$ ), and a new image was collected every $15 \mathrm{~ms}$.

All image analysis was automated with author-written analysis algorithms written in MATLAB. $10 p$ and $10 \mathrm{~s}$ images for a given timepoint were summed to form a single $p$ and a single s image for each time point. Camera background was subtracted from each image. A region of the cell containing the nucleus was chosen by the user for automated detection and identification of NPC, to prevent the analysis algorithm from considering any cytoplasmic puncta. NPCs were identified via an automated algorithm using the Laplacian of Gaussian (LoG) algorithm [written by Vincent Garcia (Garcia,

11 2020), based on (Lindeberg, 1998)]. NPCs were excised from a background subtracted

12 image (top-hat filtered), and both polarizations were fit to a Gaussian. If either

13 polarization did not fit to a Gaussian, the data point was rejected. The intensities from

14 the original (not-top-hat filtered but camera background subtracted and summed)

15 images were then extracted for analysis by taking the intensity from the maximum intensity pixel from each punctum at each polarization. A p:s ratio was then calculated

17 for each puncta.

Microscopy: Immunofluorescence

Cells were imaged with 488 laser (laser power: 5 mW) and 594 laser (laser power: $5 \mathrm{~mW}$ ) for $200 \mathrm{~ms}$.

Cells were grown on MatTek dishes at $37 \mathrm{C}$ in $5 \% \mathrm{pCO}_{2}$. Cells were fixed with $4 \%$ (wt/vol) paraformaldehyde in PBS for 15 minutes at room temperature. They were then washed 3 times for 5 minutes in PBS, and then permeabilized with $0.1 \%$ (vol/vol) Triton- 
1 x100 in PBS for 10 minutes. Cells were then blocked for 1 hour in blocking buffer $(0.1 \%$ vol/vol) Triton-x, 2.5\% normal donkey serum, $2.5 \%$ normal goat serum, and $1 \%$ BSA (all from Sigma). Primary antibodies were then added in blocking buffer and incubated overnight at $4 \mathrm{C}$ in a humid chamber. Cells were then washed 3 times for 5 minutes in PBS and then incubated with secondary antibody in $0.1 \%$ (vol/vol) Triton-x in PBS for 1$2 \mathrm{hr}$ at room temperature. Dishes were dried and mounted with SlowFade Diamond Antifade Mountant (Invitrogen, Waltham, MA). Antibodies used: monoclonal rat-akaryopherin a1/6 (2D9, Santa Cruz, Santa Cruz, CA, RRID:AB_2133549) at 1:500, monoclonal mouse- $\alpha-k a p 1 \beta / i m p \beta-1$ (3E9, Abcam, Cambride, MA, RRID: AB_2133989) at 1:1000, goat $\alpha$-rat (AF488, RRID:AB_2534074) at 1:2000, goat $\alpha$-mouse (AF594, RRID:AB_141372) from Invitrogen (1:2000). Images were quantified using FIJI software

12 (National Institutes of Health, Bethesda, MD) (Schindelin et al., 2012). The nuclear rim was defined as a region of interest by thresholding the fluorescent image of karyopherin $1 \beta$ (Kap1 $\beta$ ) and converting into a binary image. The image was then used to form a mask by: filling holes, eroding, outlining, and dilating. This mask was then used to

16 quantify intensity from Kap1 $\beta$ and Kap1 $\alpha$ channels.

\section{Microscopy: Light Activated Nuclear Shuttle (LANS)}

Cells were imaged every $20 \mathrm{~s}$ with 594 laser (laser power: $5 \mathrm{~mW}, 200 \mathrm{~ms}$ exposure time) for 2 minutes, then 594 laser acquisitions (laser power: $5 \mathrm{~mW}, 200 \mathrm{~ms}$ exposure time) were interleaved with pulses of 488 acquisitions (laser power: $3 \mathrm{~mW}, 2 \mathrm{~s}$ exposure time) every $20 \mathrm{~s}$ for 13 minutes, and then cells were imaged every $20 \mathrm{~s}$ with 594 excitation light (laser power: $5 \mathrm{~mW}, 200 \mathrm{~ms}$ exposure time) for 15 minutes with no 488 excitations. All excitations were done in a 'semi'-TIRF excitation mode, which restricts 
1 fluorescence to a few micrometers near to the coverslip to illuminate more of the cytosol. Regions of the nucleus and the cytosol were manually selected in FIJI (Schindelin et al., 2012), avoiding fluorescent aggregates, and quantified for

4 determination of the nuclear fluorescence/cytoplasmic fluorescence overtime. Microscopy: FRET

Cells were seeded onto fibronectin-coated MatTek dishes as described above. For starved condition, cells were grown 1 day then washed $3 x$ with PBS and placed in 1x Hank's Balanced Salt Solution with calcium and magnesium (HBSS, Gibco) for $24 \mathrm{~h}$ and imaged. For control condition, cells were grown 2 days then placed in cell imaging media as described above. Cells were imaged with the 488 laser (laser power: $15 \mathrm{~mW}$,

$11200 \mathrm{~ms}$ exposure time) and the 594 laser (laser power: $30 \mathrm{~mW}, 200 \mathrm{~ms}$ exposure time).

12 The mCherry was then bleached by imaging every second with the 594 laser (laser

13 power: $30 \mathrm{~mW}, 950 \mathrm{~ms}$ exposure time) for 1 minute. Cells were then imaged again with

14 the 488 laser (laser power: $15 \mathrm{~mW}, 200 \mathrm{~ms}$ exposure time) and the 594 laser (laser

15 power: $30 \mathrm{~mW}, 200 \mathrm{~ms}$ exposure time).

16

\section{FRET Quantification}

NPCs are manually identified in a pre-bleached images in Metamorph and then tracked to a post-bleach image. The maximum value pixel is quantified. FRET values are calculated as:

$$
\text { FRET }_{\text {eff }}=\frac{\left(I_{\text {post }}-I_{\text {pre }}\right)}{I_{\text {post }}}
$$




\section{Transfections}

Nups and LANS constructs were transfected 48 hours before imaging, with Fugene6 (Promega, Madison, WI). Kap1-mCherry and DN-Ran were transfected 16 hours before imaging. Cells were transfected with $1 \mu \mathrm{g}$ DNA (for LANS, Nup133mEGFP, and Kap1-mCherry constructs), 500 ng DNA (BFP-DNRan constructs, Nup93mEGFP), or 250 ng DNA (Nup54-mEGFP) and $3 \mu$ L FuGENE6 (Promega) in Opti-Mem I (Gibco) to a final volume of $100 \mu \mathrm{L}$ according to the manufacturer's instructions.

\section{Digitonin Permeabilization}

Cells were permeabilized as previously described (Adam et al., 1992). Cells were incubated on ice for $5 \mathrm{~min}$. Then cells were washed in cold transport buffer (TB) ( $20 \mathrm{mM}$ HEPES, $110 \mathrm{mM}$ potassium acetate, $5 \mathrm{mM}$ sodium acetate, $2 \mathrm{mM}$ magnesium acetate, and $1 \mathrm{mM}$ ethylene glycol tetraacetic acid (EGTA) at pH 7.3). Cells were then incubated on ice in TB with $34 \mathrm{ug} / \mathrm{mL}$ digitonin (Sigma) for 5 minutes. Cells were then washed twice in cold TB and twice in 37C TB with 1.5\% (wt/vol) 360kD polyvinylpyrrolidone (PVP, Sigma). Cells were imaged in 37C TB with 1.5\% PVP. In order to confirm that 34 $\mu \mathrm{g} / \mathrm{mL}$ digitonin left the nuclear envelope intact but resulted in the permeabilization of the plasma membrane, cells were incubated with $500 \mathrm{ng} / \mathrm{mL}$ R-phycoerythrin (ThermoFisher, Waltham, MA) for 10 minutes and then imaged. R-phycoerythrin is a $240 \mathrm{kDa}$, so it should not readily diffuse through the NPC and can be used as a marker for nuclear integrity.

\section{Protein Purification}

His-tagged Ran in the pET28 vector (a gift from Dr. Günter Blobel) was transformed into BL21 (DE3) RIL-competent cells (Stratagene, St. Louis, MO). Expression was 
$1 \quad$ induced with $0.5 \mathrm{mM}$ isopropyl $\beta$-D-1-thiogalactopyranoside (IPTG, Sigma) and cells were grown for 3 hours at $37 \mathrm{C}$. Cells were spun at $6,000 \times \mathrm{g}$ for $10 \mathrm{~min}$ at $4 \mathrm{C}$ (Sorvall SLC-6000, Waltham, MA) and the pellet was frozen overnight. The pellet was resuspended in $50 \mathrm{mM}$ TRIS pH8, $150 \mathrm{mM} \mathrm{NaCl}$ with benzonase endonuclease (at 25 $\mathrm{U} / \mathrm{mL}$, Millipore, Waltham, MA) and rLysozyme (at $12 \mathrm{U} / \mathrm{mL}$, Millipore) and 1x EDTA-

6 free cOmplete protease inhibitors (Roche, Waltham, MA). The resuspended pellet was passed through a high-pressure homogenizer (Avestin EmuliFlex-C3, ATA Scientific, Taren Point, Australia) for lysis. The lysate was spun at $30,600 \times \mathrm{g}$ at $4 \mathrm{C}$ for $30 \mathrm{~min}$

9 (Sorvall SS-34 rotor). Imidazole was added to the lysate to a concentration of $10 \mathrm{mM}$.

10 The supernatant was added to Ni-NTA beads (Qiagen, Germantown, MD) and nutated

11 at $4 \mathrm{C}$ for $1.5-2$ hours. This supernatant was then loaded on a column (Qiagen) and washed 6x with $50 \mathrm{mM}$ Tris pH8, $150 \mathrm{mM} \mathrm{NaCl}, 20 \mathrm{mM}$ imidazole. Ran was then eluted

13 with $50 \mathrm{mM}$ TRIS pH8, $150 \mathrm{mM} \mathrm{NaCl}, 300 \mathrm{mM}$ imidazole in $500 \mu \mathrm{L}$ fractions. The

14 concentration of Ran was estimated by $\mathrm{OD}_{600}$ and the fractions with the highest

15 concentrations were pooled, buffer exchanged, and concentrated on Amicon Ultra

16 Centrifugal Filter (10KD cutoff, Millipore). Samples were stored in aliquots of $10 \mathrm{mg}$ in

$1750 \mathrm{mM}$ TRIS pH, $150 \mathrm{mM} \mathrm{NaCl}$, and $10 \%$ glycerol at $-80 \mathrm{C}$. Purification was confirmed by running the protein on a $4-12 \%$ Bis-Tris gel (Novex, Waltham, MA) and performing a Coomassie (PageBlue, Thermo Scientific). with $0.3 \mathrm{mM}$ IPTG. Kap1 $\alpha$ was grown overnight ( 16 hours) at 18C following IPTG

23 induction. Kap1 $\alpha$ was stored in $200 \mu$ M aliquots, Kap1 $\beta$ in $20 \mu \mathrm{M}$ aliquots, and NTF2 in 
1 aliquots of $11.4 \mathrm{mg} / \mathrm{ml}$. The SV40 NLS was cloned into pNCStdTomato (a gift from Erik Rodriguez \& Roger Tsien (Addgene plasmid \#91767; http://n2t.net/addgene:91767; RRID:Addgene_91767). This protein was purified as above except it was grown overnight and no IPTG was added, because expression is constitutive.

$\underline{\text { Cargo Translocation }}$ was performed. Cells were permeabilized as described above by treating with digitonin and subsequently removing cytosol by gentle washing. Cells were then incubated for 30 minutes at 37C with a transport mix. We tested two conditions: +Kaps and -Kaps. Both conditions contained the following base mix: $1.5 \mu \mathrm{M}$ NLS-tdTomato, $0.1 \mathrm{mM}$ GTP

11 (ThermoFischer Scientific), $2 \mu \mathrm{M}$ Ran, and $1 \mu \mathrm{M}$ NTF2 in TB + pvp as described above. Both conditions also contained an ATP regenerating system, which includes $1 \mathrm{mM}$ ATP

13 (VWR), $1 \mathrm{mg} / \mathrm{ml}$ creatine phosphate (Sigma), and $15 \mathrm{U} / \mathrm{ml}$ creatine phosphokinase (Millipore). Finally, the two conditions either did or did not contain a receptor mix (+/-

15 Kaps), consisting of $1.5 \mu \mathrm{M}$ Kap $1 \alpha$ and $1 \mu \mathrm{M}$ Kap1 $\beta$. cargo and transport elements from the nuclear periphery. Cells were incubated with live cell Hoechst (NucBlue Live ReadyProbes, Hoeschst 33342, ThermoFischer Scientific). Cells were then imaged to quantify the nuclear NLS-tdTomato. All excitations were done in a 'semi'-TIRF excitation mode, which restricts fluorescence to a few micrometers near to the coverslip to illuminate more of the nucleoplasm. Nuclear regions were determined by constructing a mask in FIJI using the Hoescht staining and finding the average intensity in arbitrary units of the tdTomato signal (Schindelin et al., 2012). 


\section{$\underline{\text { Ran GTP Loading and Karyopherin Removal }}$}

Ran was loaded with GDP using published methods (Lowe et al., 2015). 1 mM Ran was incubated with $50 \mathrm{mM}$ GDP in $10 \mathrm{mM}$ HEPES pH 7.3, $100 \mathrm{mM} \mathrm{NaCl}$ and $10 \mathrm{mM}$ EDTA at RT for 30 min. Then the sample was diluted $2.5 \mathrm{x}$ and the reaction was stopped to a final concentration of $25 \mathrm{mM} \mathrm{MgCl} 2$ added in four portions in 1 min intervals and was dialyzed in TB overnight.

To remove kaps, cells were treated with Ran mix using published methods (Adam et al., 1992; Kapinos et al., 2017). Cells were incubated with Ran mix in TB+pvp for 1 hour (2 mM GTP, $0.1 \mathrm{mM} \mathrm{ATP,} 4 \mathrm{mM}$ creatine phosphate, $20 \mathrm{U} / \mathrm{mL}$ creatine kinase, $5 \mathrm{M}$ RanGDP, $4 \mu \mathrm{M}$ NTF2, and $1 \mathrm{mM}$ DTT).

\section{CRISPR Cell Lines}

Guides and repair templates were constructed by using the Benchling CRISPR design tool (Benchling, San Francisco, CA). Guides were cloned into the pSpCas9(BB)2A-Puro (PX459) V2.0 plasmid following standard protocols as developed by the Zhang lab (Ran et al., 2013). The repair templates (for Nup54-mEGFP(0) and Nup133mEGFP(-8) were synthesized by GenScript as dsDNA. This DNA was then A'-tailed and placed in a TOPO-TA Cloning Vector (2.1-TOPO, ThermoFisher Scientific). Repair templates for Nup54-mEGFP(1) and Nup-mEGFP(2) and Nup133-mEGFP(-7) were cloned from the repair template plasmids constructed above via the QuikChange Lightning Site-Directed Mutagenesis Kit (Agilent, Santa Clara, CA). For each CRISPR cell line, cells were transfected with linearized repair templates and the PX459 plasmid containing the appropriate guides using FuGENE HD (Promega) with a ratio of Reagent : DNA of $3: 1$ according to manufacturer's protocol. Transfected cells were selected via 
1 antibiotic selection with puromycin $(0.6 \mu \mathrm{g} / \mathrm{mL}$, Invivogen, San Diego, CA) for 48 hours.

2 Cells were released from antibiotic selection and allowed to recover for 24 hours. GFP

3 positive cells were collected in the Rockefeller University Flow Cytometry Resource

4 Center using a FACSAria II flow cytometer (BD Biosciences, San Jose, CA). Cells were resuspended in PBS (no calcium/no magnesium, Gibco), 0.5\% (vol/vol) bovine serum

6 albumin (BSA, Sigma), 5mM EDTA (Gibco), and 15 mM HEPES (ThermoFisher). GFP

7 positive cells were sorted and screened via live cell microscopy for signal at the nuclear

$8 \quad \operatorname{rim}(\sim 95 \%$ of GFP positive cells showed nuclear rim GFP signal). Cell lines were

9 screened for homozygosity via PCR. Two bands were seen for heterozygotes and one

10 band for homozygotes, both fragments were sequenced to confirm amplification of

11 correct region and proper incorporation of the mEGFP. In order to confirm that the

12 mEGFP was not incorporated elsewhere and producing another labeled protein within

13 our cells, western blots were performed. Protein samples were run on a 4-12\% Bis-Tris

14 gel (Novex) and Western blots were performed with the following antibodies: $\alpha$-GFP

15 (Clontech Living Colors 632381 (JL-8); RRID:AB_2313808, mouse monoclonal,

$161: 1,000)$ and goat $\alpha-m o u s e-H R P$ (Sigma-Aldrich; RRID:AB_258476, 1:50,000).

17 Membranes were then striped with Restore ${ }^{\mathrm{TM}}$ Western Blot Stripping Buffer

18 (ThermoFisher) and western blots were performed with the following antibodies: $\alpha$-actin

19 (Abcam; RRID:AB_476743, 1:1,000) and goat a-mouse-HRP (Sigma-Aldrich;

20 RRID:AB_258476, 1:50,000). All blocking and incubations were done in 5\% (wt/vol)

21 nonfat milk powder in Tris-buffered saline with Tween 20.

22 Quantification and Statistical Analysis 
bioRxiv preprint doi: https://doi.org/10.1101/2020.07.27.223081; this version posted July 28, 2020. The copyright holder for this preprint (which

was not certified by peer review) is the author/funder, who has granted bioRxiv a license to display the preprint in perpetuity. It is made available under aCC-BY 4.0 International license.

1 Statistical analyses were performed using MATLAB Verison 2019b and are described in

2 the figure legends and in the Method Details.

3

4 


\section{Acknowledgments:}

2 We thank Günter Blobel, Elias Coutavas, and Ivo Melčák for Nup58, Kap-Alpha1, and

3 Kap-Beta1 cDNA and for the his-Ran plasmid. pSpCas9(BB)-2A-Puro (PX459) V2.0

4 was a gift from Feng Zhang (Addgene plasmid \# 62988). DN-RanQ69L was a gift from

5 Jay Brenman (Addgene plasmid \# 30309) and pTriEx-mCherry::LANS4 was a gift from

6 Brian Kuhlman (Addgene plasmid \# 60785). We thank Günter Blobel, Philip Coffino and

7 Elias Coutavas for helpful comments. Funding: The Howard Hughes Medical Institute

8 Gilliam Fellowship (J. P.).

10 Competing Interests:

11 The authors declare no competing interests. 


\section{1}

\section{References:}

Adam, S. A., Sterne-Marr, R., \& Gerace, L. (1992). Nuclear protein import using digitonin-permeabilized cells. Methods Enzymol, 219, 97-110. https://doi.org/10.1016/0076-6879(92)19013-v

Alber, F., Dokudovskaya, S., Veenhoff, L. M., Zhang, W., Kipper, J., Devos, D., Suprapto, A., Karni-Schmidt, O., Williams, R., Chait, B. T., Sali, A., \& Rout, M. P. (2007). The molecular architecture of the nuclear pore complex. Nature, 450(7170), 695. https://doi.org/10.1038/nature06405 PMID - 18046406

Atkinson, C. E., Mattheyses, A. L., Kampmann, M., \& Simon, S. M. (2013, Jan 8). Conserved spatial organization of FG domains in the nuclear pore complex. Biophysical Journal, 104(1), 37-50. https://doi.org/10.1016/j.bpj.2012.11.3823

Bischoff, F. R., Klebe, C., Kretschmer, J., Wittinghofer, A., \& Ponstingl, H. (1994, Mar 29). RanGAP1 induces GTPase activity of nuclear Ras-related Ran. Proc Natl Acad Sci U S A, 91(7), 2587-2591. https://doi.org/10.1073/pnas.91.7.2587

Bui, K. H., von Appen, A., DiGuilio, A. L., Ori, A., Sparks, L., Mackmull, M. T., Bock, T., Hagen, W., AndresPons, A., Glavy, J. S., \& Beck, M. (2013, Dec 5). Integrated structural analysis of the human nuclear pore complex scaffold. Cell, 155(6), 1233-1243. https://doi.org/10.1016/j.cell.2013.10.055

D'Angelo, M. A., Gomez-Cavazos, J. S., Mei, A., Lackner, D. H., \& Hetzer, M. W. (2012, Feb 14). A change in nuclear pore complex composition regulates cell differentiation. Developmental cell, 22(2), 446-458. https://doi.org/10.1016/j.devcel.2011.11.021

Eibauer, M., Pellanda, M., Turgay, Y., Dubrovsky, A., Wild, A., \& Medalia, O. (2015, Jun 26). Structure and gating of the nuclear pore complex. Nat Commun, 6, 7532. https://doi.org/10.1038/ncomms8532 
Garcia, V. (2020). Keypoint Extraction. Retrieved September 1 from

https://www.mathworks.com/matlabcentral/fileexchange/17894-keypoint-extraction

Hoelz, A., Debler, E. W., \& Blobel, G. (2011). The structure of the nuclear pore complex. Annu Rev Biochem, 80, 613-643. https://doi.org/10.1146/annurev-biochem-060109-151030

Johnson, D. S., Bleck, M., \& Simon, S. M. (2018, Jul 4). Timing of ESCRT-III protein recruitment and membrane scission during HIV-1 assembly. Elife, 7. https://doi.org/10.7554/eLife.36221

Kampmann, M., Atkinson, C. E., Mattheyses, A. L., \& Simon, S. M. (2011, Jun). Mapping the orientation of nuclear

Kapinos, L. E., Huang, B., Rencurel, C., \& Lim, R. Y. H. (2017, Nov 6). Karyopherins regulate nuclear pore complex barrier and transport function. J Cell Biol, 216(11), 3609-3624.

21 https://doi.org/10.1083/jcb.201702092

Johnson, D. S., Toledo-Crow, R., Mattheyses, A. L., \& Simon, S. M. (2014, Mar 4). Polarization-controlled TIRFM with focal drift and spatial field intensity correction. Biophys $J, 106(5), 1008-1019$. https://doi.org/10.1016/j.bpj.2013.12.043 pore proteins in living cells with polarized fluorescence microscopy. Nature Structural \& Molecular Biology, 18(6), 643-649. https://doi.org/10.1038/nsmb.2056

Kim, S. J., Fernandez-Martinez, J., Nudelman, I., Shi, Y., Zhang, W., Raveh, B., Herricks, T., Slaughter, B. D., Hogan, J. A., Upla, P., Chemmama, I. E., Pellarin, R., Echeverria, I., Shivaraju, M., Chaudhury, A. S., Wang, J., Williams, R., Unruh, J. R., Greenberg, C. H., Jacobs, E. Y., Yu, Z., de la Cruz, M. J., Mironska, R., Stokes, D. L., Aitchison, J. D., Jarrold, M. F., Gerton, J. L., Ludtke, S. J., Akey, C. W., Chait, B. T., Sali, A., \& Rout, M. P. (2018, Mar 22). Integrative structure and functional anatomy of a nuclear pore complex. Nature, 555(7697), 475-482. https://doi.org/10.1038/nature26003 
Kimura, M., Morinaka, Y., Imai, K., Kose, S., Horton, P., \& Imamoto, N. (2017). Extensive cargo identification reveals distinct biological roles of the 12 importin pathways. eLife, 6. https://doi.org/10.7554/elife.21184

Kosinski, J., Mosalaganti, S., von Appen, A., Teimer, R., DiGuilio, A. L., Wan, W., Bui, K. H., Hagen, W. J., Briggs, J. A., Glavy, J. S., Hurt, E., \& Beck, M. (2016, Apr 15). Molecular architecture of the inner ring scaffold of the human nuclear pore complex. Science, 352(6283), 363-365. https://doi.org/10.1126/science.aaf0643

Kudo, N., Matsumori, N., Taoka, H., Fujiwara, D., Schreiner, E. P., Wolff, B., Yoshida, M., \& Horinouchi, S. (1999, Aug 3). Leptomycin B inactivates CRM1/exportin 1 by covalent modification at a cysteine residue in the central conserved region. Proc Natl Acad Sci U S A, 96(16), 9112-9117. https://doi.org/10.1073/pnas.96.16.9112

Lindeberg, T. (1998, November 01). Feature Detection with Automatic Scale Selection [journal article]. International Journal of Computer Vision, 30(2), 79-116. https://doi.org/10.1023/a:1008045108935

Lowe, A. R., Tang, J. H., Yassif, J., Graf, M., Huang, W. Y., Groves, J. T., Weis, K., \& Liphardt, J. T. (2015, Mar 6). Importin-beta modulates the permeability of the nuclear pore complex in a Ran-dependent manner. Elife, 4. https://doi.org/10.7554/eLife.04052

Lupu, F., Alves, A., Anderson, K., Doye, V., \& Lacy, E. (2008, Jun). Nuclear pore composition regulates neural stem/progenitor cell differentiation in the mouse embryo. Developmental cell, 14(6), 831-842. https://doi.org/10.1016/j.devcel.2008.03.011

Mahamid, J., Pfeffer, S., Schaffer, M., Villa, E., Danev, R., Cuellar, L. K., Forster, F., Hyman, A. A., Plitzko, J. M., \& Baumeister, W. (2016, Feb 26). Visualizing the molecular sociology at the HeLa cell nuclear periphery. Science, 351(6276), 969-972. https://doi.org/10.1126/science.aad8857 
Mattheyses, A. L., Kampmann, M., Atkinson, C. E., \& Simon, S. M. (2010). Fluorescence Anisotropy Reveals Order and Disorder of Protein Domains in the Nuclear Pore Complex. Biophysical Journal, 99(6), 17061717. https://doi.org/10.1016/j.bpj.2010.06.075 PMID - 20858414

Melcak, I., Hoelz, A., \& Blobel, G. (2007, Mar 23). Structure of Nup58/45 suggests flexible nuclear pore diameter by intermolecular sliding. Science, 315(5819), 1729-1732. https://doi.org/10.1126/science.1135730

Moore, M. S., \& Blobel, G. (1993, Oct 14). The GTP-binding protein Ran/TC4 is required for protein import into the nucleus. Nature, 365(6447), 661-663. https://doi.org/10.1038/365661a0

Mosalaganti, S., Kosinski, J., Albert, S., Schaffer, M., Strenkert, D., Salomé, P. A., Merchant, S. S., Plitzko, J. M.,

Ori, A., Banterle, N., Iskar, M., Andrés-Pons, A., Escher, C., Bui, H. K., Sparks, L., Solis-Mezarino, V., Rinner, O., Bork, P., Lemke, E. A., \& Beck, M. (2013). Cell type-specific nuclear pores: a case in point for contextdependent stoichiometry of molecular machines. Molecular Systems Biology, 9(1), 648.

Pumroy, R. A., \& Cingolani, G. (2015, Feb 15). Diversification of importin-alpha isoforms in cellular trafficking and disease states. Biochem J, 466(1), 13-28. https://doi.org/10.1042/BJ20141186 https://doi.org/10.1038/msb.2013.4 PMID - 23511206 
Reichelt, R., Holzenburg, A., Buhle, E. L., Jarnik, M., Engel, A., \& Aebi, U. (1990). Correlation between structure and mass distribution of the nuclear pore complex and of distinct pore complex components. The Journal of Cell Biology, 110(4), 883-894. https://doi.org/10.1083/jcb.110.4.883 PMID - 2324201

Rout, M. P., \& Blobel, G. (1993). Isolation of the yeast nuclear pore complex. The Journal of Cell Biology, 123(4), 771-783. https://doi.org/10.1083/jcb.123.4.771

Schindelin, J., Arganda-Carreras, I., Frise, E., Kaynig, V., Longair, M., Pietzsch, T., Preibisch, S., Rueden, C.,

Selles, J., Penrad-Mobayed, M., Guillaume, C., Fuger, A., Auvray, L., Faklaris, O., \& Montel, F. (2017, Nov 7). Nuclear pore complex plasticity during developmental process as revealed by super-resolution microscopy.

16 Sci Rep, 7(1), 14732. https://doi.org/10.1038/s41598-017-15433-2

Sharma, A., Solmaz, S. R., Blobel, G., \& Melcak, I. (2015, Jul 24). Ordered Regions of Channel Nucleoporins Nup62, Nup54, and Nup58 Form Dynamic Complexes in Solution. J Biol Chem, 290(30), 18370-18378. https://doi.org/10.1074/jbc.M115.663500

Solmaz, S. R., Chauhan, R., Blobel, G., \& Melcak, I. (2011, Oct 28). Molecular architecture of the transport channel of the nuclear pore complex. Cell, 147(3), 590-602. https://doi.org/10.1016/j.cell.2011.09.034 
Stochaj, U., Rassadi, R., \& Chiu, J. (2000, Nov). Stress-mediated inhibition of the classical nuclear protein import pathway and nuclear accumulation of the small GTPase Gsp1p. FASEB J, 14(14), 2130-2132. https://doi.org/10.1096/fj.99-0751fje

Vollmer, B., \& Antonin, W. (2014, May). The diverse roles of the Nup93/Nic96 complex proteins - structural scaffolds of the nuclear pore complex with additional cellular functions. Biol Chem, 395(5), 515-528. https://doi.org/10.1515/hsz-2013-0285

8

Yumerefendi, H., Dickinson, D. J., Wang, H., Zimmerman, S. P., Bear, J. E., Goldstein, B., Hahn, K., \& Kuhlman, B. (2015). Control of Protein Activity and Cell Fate Specification via Light-Mediated Nuclear Translocation. PLOS ONE, 10(6), e0128443. https://doi.org/10.1371/journal.pone.0128443 PMID -

13

14 Zwerger, M., Eibauer, M., \& Medalia, O. (2016). Insights into the gate of the nuclear pore complex. Nucleus, 7(1), 
SUPPLEMENTAL INFORMATION:

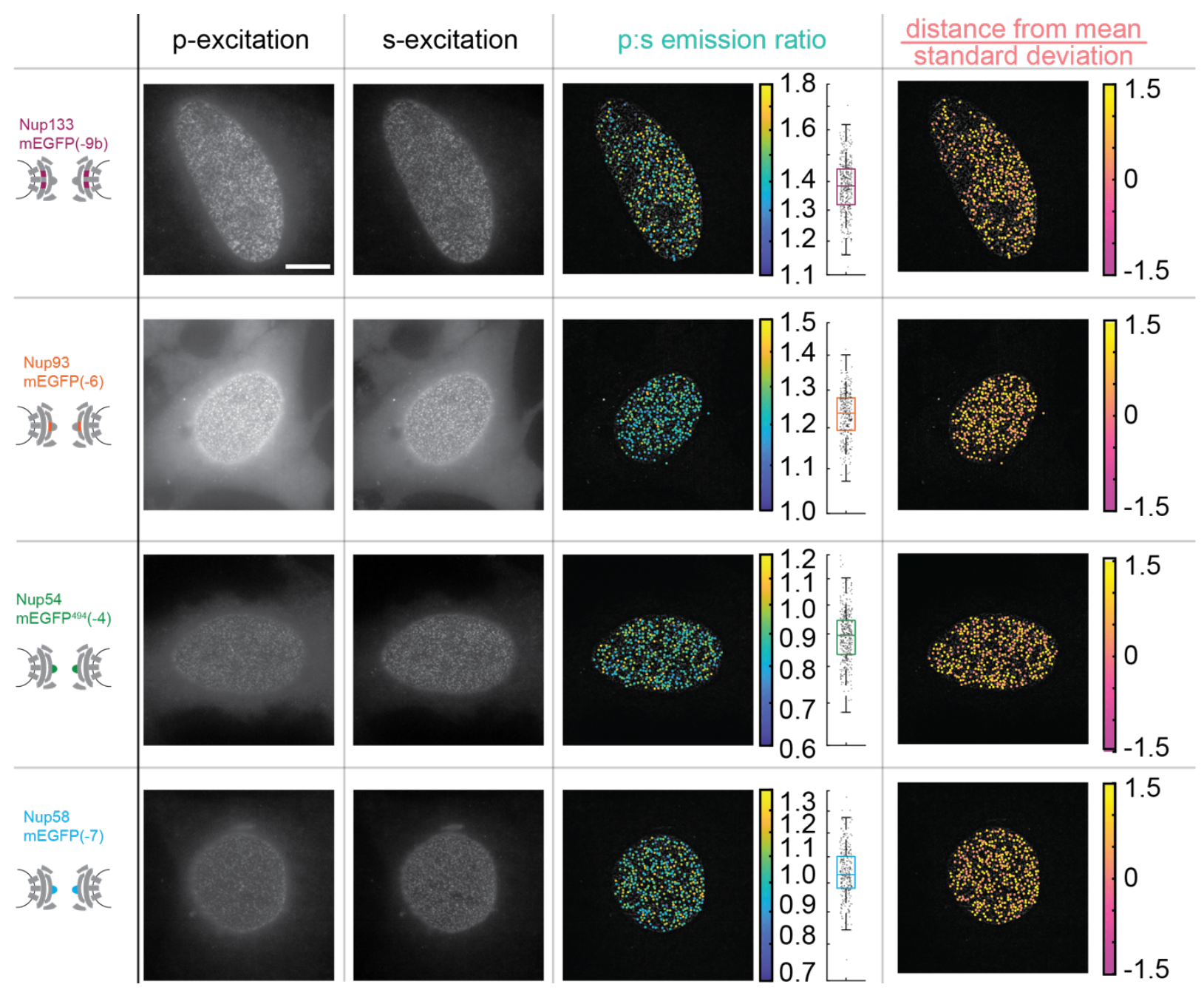

Figure 1-figure supplement 1. The Orientation of Nup-mEGFP Fusion Proteins

Four different Nup-mEGFP (Nup133, Nup93, Nup54, and Nup58) were transiently

expressed for 48 hours in HeLa cells. (Columns 1-2) The constructs were alternately

excited with $p$-polarized (perpendicular to the coverslip) and $s$-polarized (parallel to the coverslip) light $($ Scale bar $=10 \mu \mathrm{m})$. (Column 3$)$ The p:s ratios, a measurement of

9 orientation of mEGFP relative to the coverslip are calculated for each NPC. The color of

10 the dot represents the p:s ratio of the NPC upon which it is superimposed. The box plot 
1 represents the data directly taken from the cell next to it. Each dot represents a single

2 NPC measured. (Column 4) The difference from the mean divided by the standard

3 deviation is shown in the final column, illustrating how the p:s ratio does not vary with

4 respect to the basal surface of the nucleus and that the cell holds its nuclear envelope

5 parallel to the coverslip.

6 


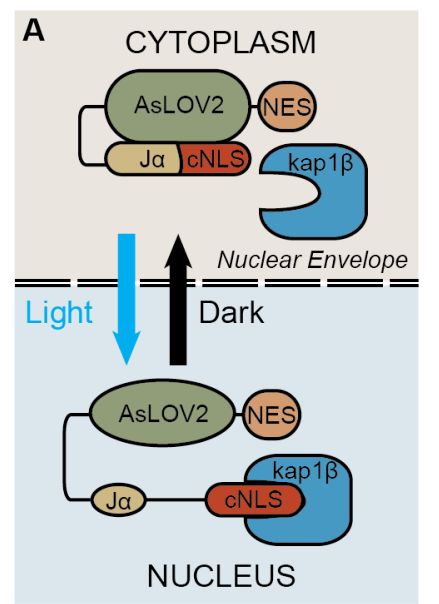

B
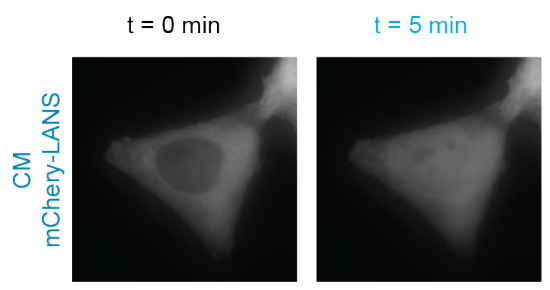

$\mathrm{t}=15 \mathrm{~min}$
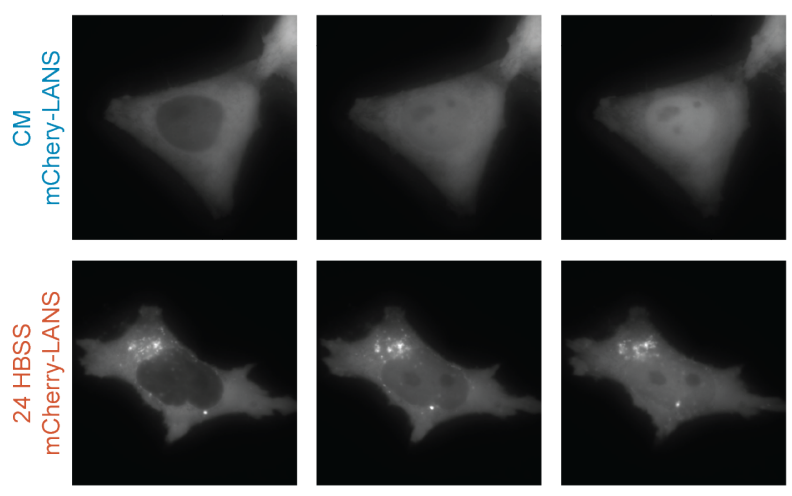

D

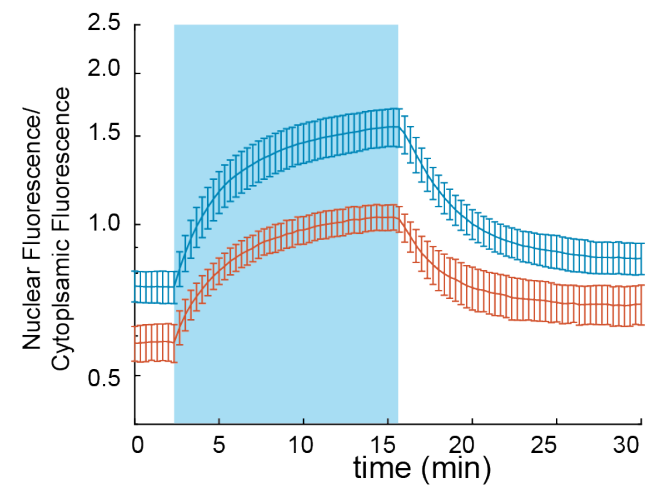

C

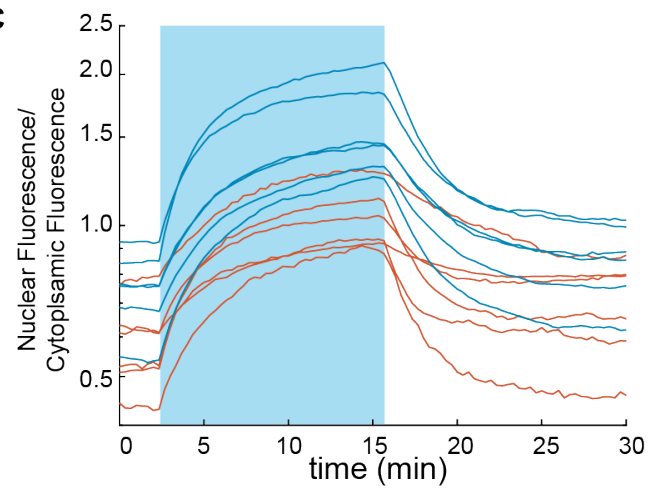

$\mathrm{t}=30 \mathrm{~min}$
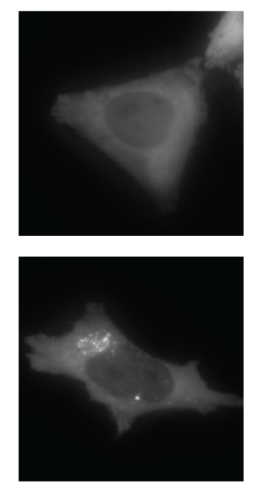

Figure 3-figure supplement 1. Nuclear Transport is Attenuated Post-Starvation.

A) The NLS domain of the LANS construct normally masked by the AsLOV2 domain and the protein remains predominantly in the cytosol. Upon stimulation with blue light, the NLS is unmasked and the construct translocates into the nucleus. Figure Adapted from (Yumerefendi et al., 2015). B) Representative images for light activation and reversion for HeLa cells in complete media (CM) and HBSS for 24 hours. (Scale bar = $10 \mu \mathrm{m})$. Images are all scaled to the same color axis. C) The change in the ratio of

9 nuclear/cytoplasmic fluorescence in HeLa cells in CM (blue) and HBSS (red). The shaded region represents the time of blue light LANS activation.

D) The average change in the ratio of nuclear/cytoplasmic fluorescence in HeLa cells in CM (blue) and 
bioRxiv preprint doi: https://doi.org/10.1101/2020.07.27.223081; this version posted July 28, 2020. The copyright holder for this preprint (which was not certified by peer review) is the author/funder, who has granted bioRxiv a license to display the preprint in perpetuity. It is made available under aCC-BY 4.0 International license.

1 the time of blue light LANS activation. Note: In starved cells, cytoplasmic regions that

2 were bright, which might be autophagosomes. These regions were excluded from

3 analysis. 
CM HBSS

A

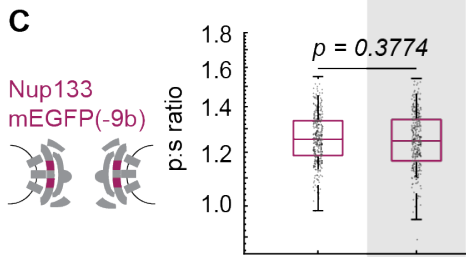

E $\operatorname{mEGFP}(-6)$

竞) ?

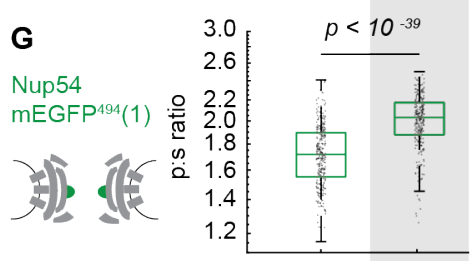

CM HBSS
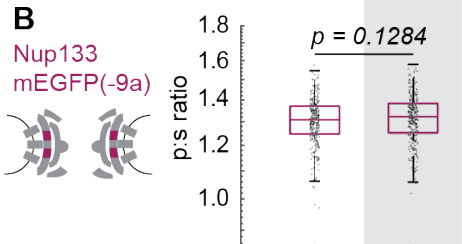

D

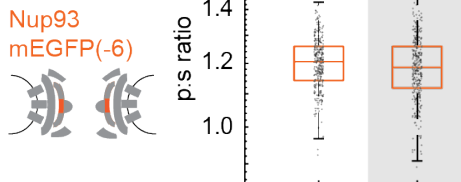

F
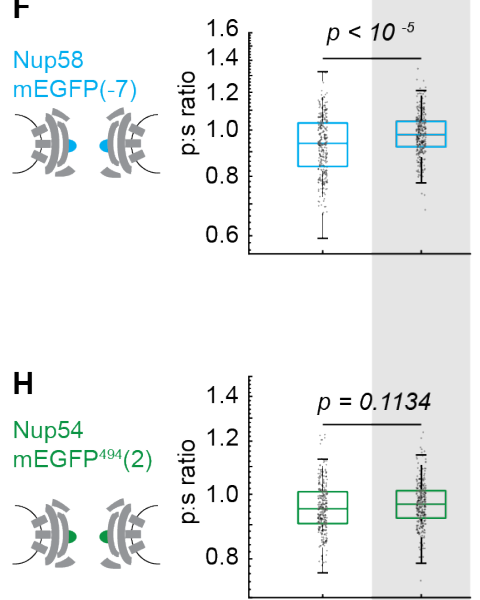

Figure 3-figure supplement 2. The Inner Ring Nups, Nup54 and Nup58, are reorganized with respect to the NPC after starvation.

Cells were maintained in complete media (CM) or starved for 24 hours in HBSS prior to imaging. The p:s ratios are presented for: (A) Nup133-mEGFP(-8b), (B) Nup133mEGFP(-9a), (C) Nup133-mEGFP(-9b), (D) Nup93-mEGFP(-6), (E) Nup58-mEGFP(-6), (F) Nup58-mEGFP(-7), (G) Nup54-mEGFP ${ }^{494}(1)$, and (H) Nup54-mEGFP ${ }^{494}(2)$. The orientation changed for Nup58-mEGFP and Nup54-mEGFP ${ }^{494}$. 
1 There was no change of orientation for Nup133-mEGFP(-8a) after starvation (Figure 3).

2 Here, the results are expanded to include Nup133-mEGFP(-8b), Nup133-mEGFP(-9a)

3 and Nup133-mEGFP(-9b), none of which show a change of orientation after starvation.

$4 \quad$ No change in orientation of Nup93-mEGFP(-5) was seen after starvation (Figure 3);

5 here, Nup93-mEGF(-6) also shows no change in orientation post-starvation.

6 Starvation shifted the orientation of Nup58-mEGFP(-8) (Figure 3), and here it shifts the

7 orientation of Nup58-mEGFP(-6) and Nup58-mEGFP(-7).

9 Starvation shifts the orientation of Nup54-mEGFP ${ }^{494}(0)$ (Figure 3), and here starvation

10 shifts the orientation of Nup54-mEGFP ${ }^{494}(1)$. A significant change in orientation is not

11 seen on Nup54-mEGFP ${ }^{494}(2)$. It is expected that the magnitude of the shift of the

12 orientation will differ between different probe locations. For example, if a starvation

13 induces a rotation of the excitation dipole of the mEGFP from $-52^{\circ}$ to $+52^{\circ}$ relative to the

14 axis normal to the coverslip, then p:s ratio will not change. This result underscores the

15 importance of testing different linker lengths. HeLa cells were imaged 48 hours post

16 transfection. ( $n=300$ NPCs, 10 cells, boxes indicate quartiles, center bars indicate

17 medians, student t-test). 

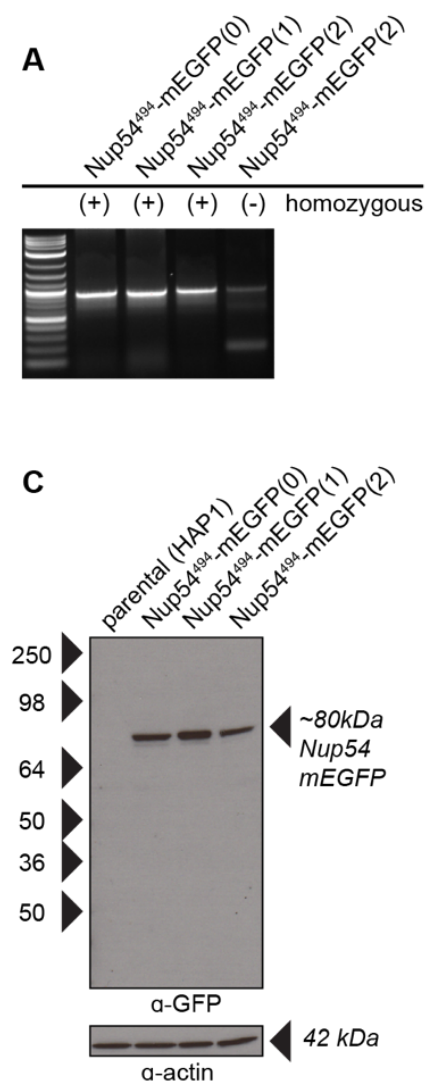

B
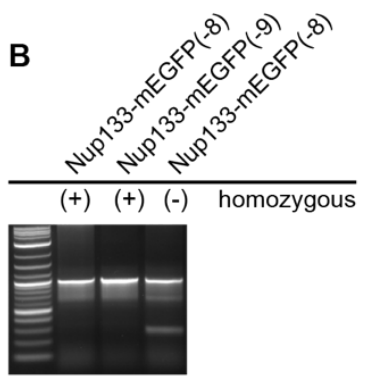

D
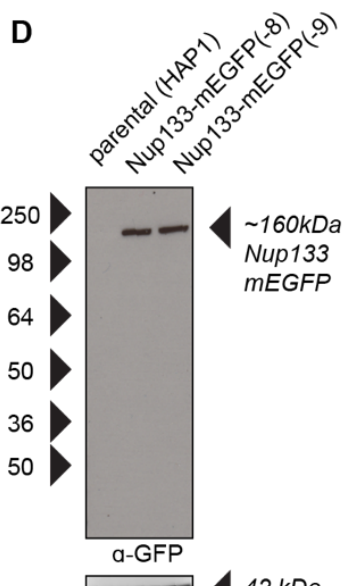

mEGFP

2 Figure 4-figure supplement 1. Validation of CRISPR Cell Lines.

A-B) PCR amplification of the Nup54 and Nup133 region reveal one band when the Nup-mEGFP region is homozygous for mEGFP incorporation and two bands when the region is heterozygous. The bands were sequenced to verify proper incorporation of the mEGFP. The validated homozygous clones were used for our experiments. C-D)

8 Western blots probed for $\alpha$-GFP detects Nup54 and Nup133 fusion proteins as expected. The blots were stripped of $\alpha$-GFP and probed with $\alpha$-actin to confirm protein

10 loading. 
1

\section{B}

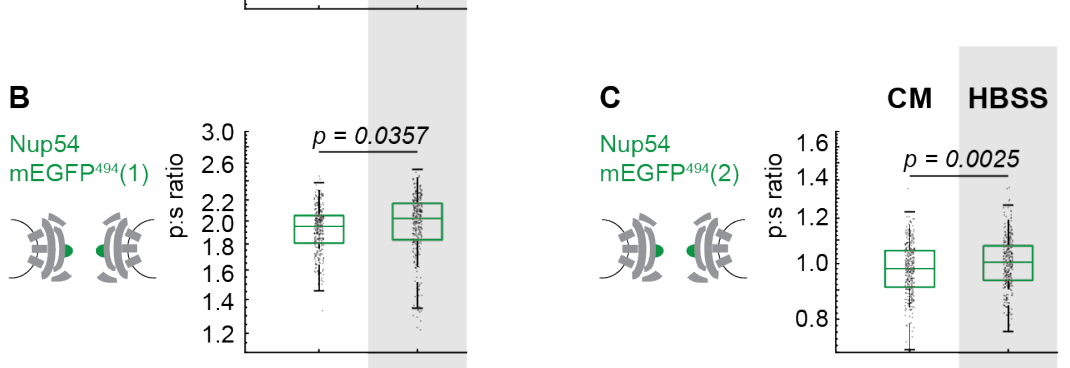

CM HBSS

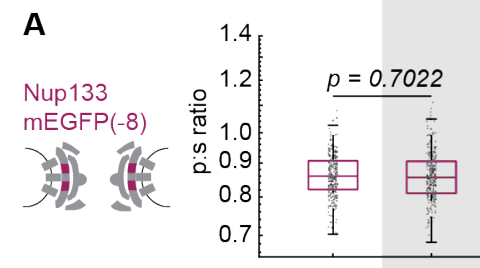

Figure 4-figure supplement 2. The Inner Ring Nup Nup54 is reorganized with

\section{respect to the NPC after starvation.}

Hap1 cell lines that had been engineered with CRISPR/Cas9 were maintained in complete media $(\mathrm{CM})$ or starved for 24 hours in HBSS prior to imaging. The p:s ratios are presented for: (A) Nup133-mEGFP(-8), (B) Nup54-mEGFP494(1), (C) Nup54-

mEGFP ${ }^{494}(2)$. These results are similar to what was observed for the transiently transfected cells in Figure 3 and Figure $3 S$ and the CRISPR line in Figure 4E. The cell

line used in Figure 4E was Nup133-mEGFP(-9), and in this figure the cell line used was Nup133-mEGFP(-8). The CRISPR cell line used in Figure 4E was Nup54-mEGFP494(0);

and in this figure, the cell lines used are Nup54-mEGFP494(1) and Nup54-mEGFP494(2).

12 ( $n=300$ NPCs, 10 cells, boxes indicate quartiles, center bars indicate medians, student 13 t-test). 
bioRxiv preprint doi: https://doi.org/10.1101/2020.07.27.223081; this version posted July 28, 2020. The copyright holder for this preprint (which

was not certified by peer review) is the author/funder, who has granted bioRxiv a license to display the preprint in perpetuity. It is made available under aCC-BY 4.0 International license.

A

Dig: $0 \mu \mathrm{g} / \mathrm{mL}$
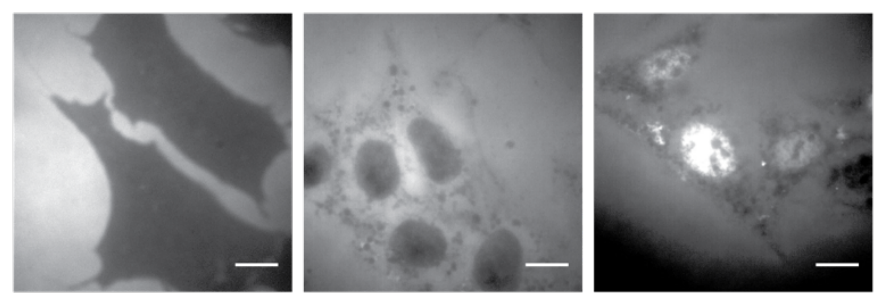

B

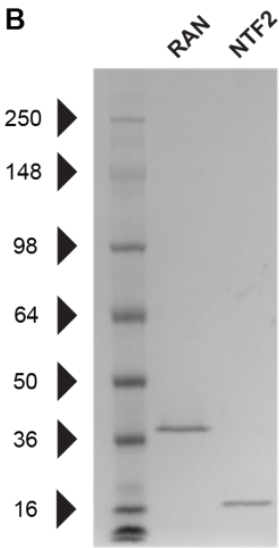

C

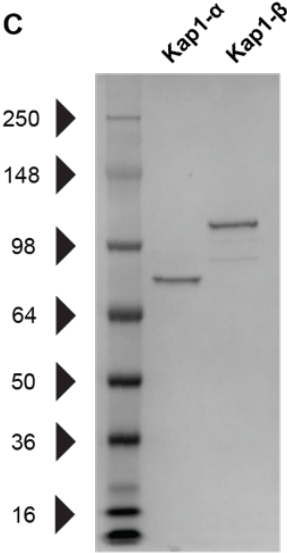

D
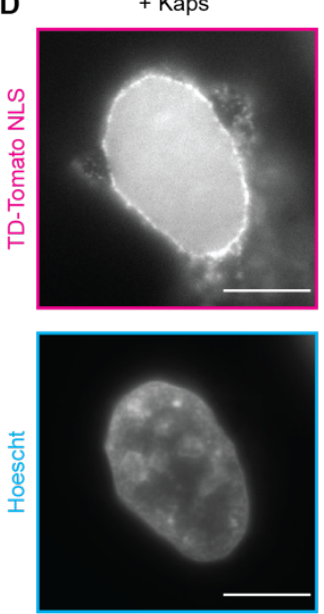

F
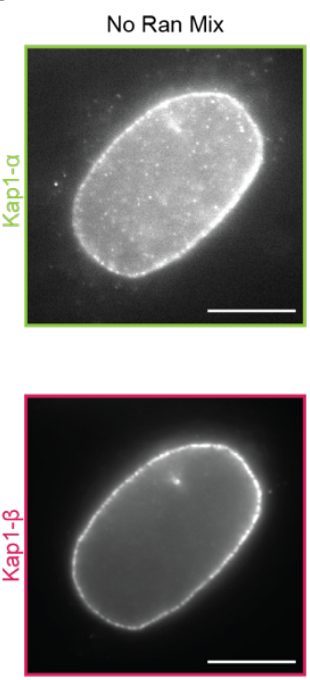

- Kaps
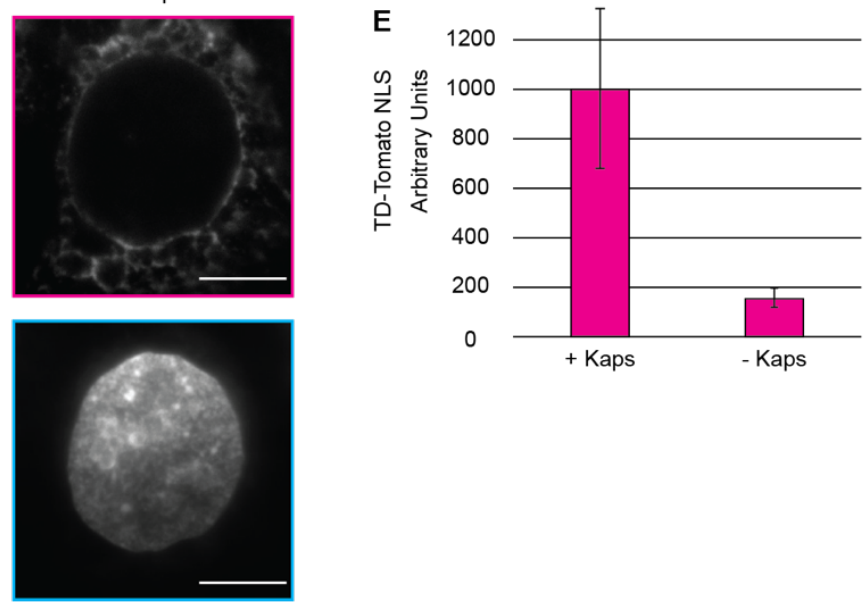

G

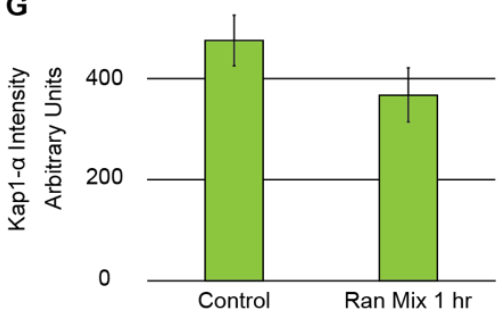

H

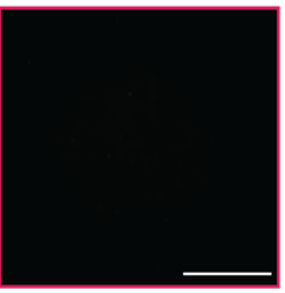

Plus Ran Mix
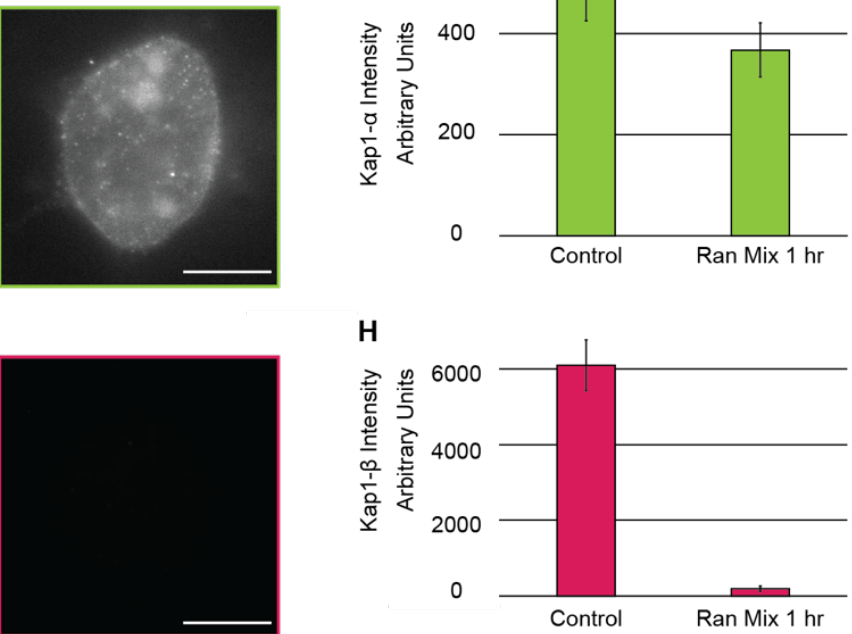
1 Figure 5-figure supplement 1. Nuclear transport factors are be introduced to nuclear periphery using digitonin permeabilization of the plasma membrane.

3 A) Cells were incubated with R-phycoerythrin after the digitonin permeabilization protocol was performed with (left) $0 \mu \mathrm{g} / \mathrm{mL}$ digitonin, for which no R-phycoerythrin is

5 detected in the cell, (middle) $34 \mu \mathrm{g} / \mathrm{mL}$, for which R-phycoerythrin has entered the

6 cytosol but is excluded from the nucleus, and (right) $100 \mu \mathrm{g} / \mathrm{mL}$ digitonin for which R-

7 phycoerythrin is seen throughout the cytosol and nucleus. (Scale Bar $=10 \mu \mathrm{m}$ ). B-C)

8 The Coomassie-stained gels show purified Ran, NTF2, Kap1- $\alpha$, Kap1- $\beta$ proteins with

9 minimal contaminants. D) Cells were incubated with an ATP-regenerating system, Ran-

10 GTP, NTF2, and an NLS-TD-Tomato cargo in the presence and absence of Kap1- $\alpha$

11 and Kap1- $\beta$. E) Amount of NLS-TD-Tomato accumulated in the nucleus was measured

12 by quantifying the average fluorescence signal in the nucleus, as defined via Hoechst

13 staining. ( $n=5$ cells, error bars represent standard deviation). F-G) Removal of kaps was

14 confirmed with quantitative IF. (Scale Bar = $10 \mu \mathrm{m}$; error bars represent standard

15 deviation; $n=5$ cells). 
1 Table S1: Nup-mEGFP Transfected Fusion Proteins

2 Size of mEGFP Deletion: Number of amino acids deleted from the amino terminus of mEGFP.

3 Net Linker Size: Number of amino acids in linker minus the deletions from Nup and mEGFP.

\begin{tabular}{|c|c|c|c|c|c|c|}
\hline Construct Name & Nup & $\begin{array}{l}\text { Position of } \\
\text { mEGFP } \\
\text { within Nup }\end{array}$ & $\begin{array}{l}\text { Size of } \\
\text { mEGFP } \\
\text { Deletio } \\
n \\
\text { (AAs) }\end{array}$ & $\begin{array}{l}\text { Net } \\
\text { Linke } \\
\text { r Size } \\
\text { (AAs) }\end{array}$ & $\begin{array}{l}\text { Amino Acid Linker } \\
\text { Sequence } \\
\text { Nup Sequence in } \\
\text { Blue } \\
\text { mEGFP Sequence } \\
\text { in Green }\end{array}$ & Figures \\
\hline $\begin{array}{l}\text { Nup133_mEGFP(- } \\
\text { 8a)* }\end{array}$ & Nup133 & $\begin{array}{l}\text { Carboxyl- } \\
\text { Terminus - } \\
2\end{array}$ & 6 & -8 & EYYVQGELFT & $\begin{array}{l}\text { Fig. } 2 A ; \\
\text { Fig. } 3 A\end{array}$ \\
\hline $\begin{array}{l}\text { Nup133_mEGFP(- } \\
\text { 8b) }\end{array}$ & Nup133 & $\begin{array}{l}\text { Carboxyl- } \\
\text { Terminus - } \\
3\end{array}$ & 5 & -8 & EYYVQEELFT & $\begin{array}{l}\text { Fig. 2A; } \\
\text { Fig. S3A }\end{array}$ \\
\hline $\begin{array}{l}\text { Nup133_mEGFP(- } \\
\text { 9a) }\end{array}$ & Nup133 & $\begin{array}{l}\text { Carboxyl- } \\
\text { Terminus - } \\
3\end{array}$ & 6 & -9 & EYYVQELFT & $\begin{array}{l}\text { Fig. 2A; } \\
\text { Fig. S3B }\end{array}$ \\
\hline $\begin{array}{l}\text { Nup133_mEGFP(- } \\
\text { 9b) }\end{array}$ & Nup133 & $\begin{array}{l}\text { Carboxyl- } \\
\text { Terminus - } \\
4\end{array}$ & 5 & -9 & EYYVEEELFT & $\begin{array}{l}\text { Fig. } 2 \mathrm{~A} ; \\
\text { Fig. S3C }\end{array}$ \\
\hline Nup93_mEGFP(-5) ${ }^{\star *}$ & Nup93 & $\begin{array}{l}\text { Carboxyl - } \\
\text { Terminus }\end{array}$ & 5 & -5 & EVLMNEELFT & $\begin{array}{l}\text { Fig. 2B; } \\
\text { Fig. 3B }\end{array}$ \\
\hline Nup93_mEGFP(-6) & Nup93 & $\begin{array}{l}\text { Carboxyl - } \\
\text { Terminus }\end{array}$ & 6 & -6 & EVLMNELFT & $\begin{array}{l}\text { Fig. 2B; } \\
\text { Fig. S3D }\end{array}$ \\
\hline Nup58_mEGFP(-6) & Nup58 & 412 & 6 & -6 & RKMFLGELFT & $\begin{array}{l}\text { Fig. 2C; } \\
\text { Fig. S3E }\end{array}$ \\
\hline Nup58_mEGFP(-7) & Nup58 & 410 & 5 & -7 & RKMFEELFT & $\begin{array}{l}\text { Fig. 2C; } \\
\text { Fig. S3F }\end{array}$ \\
\hline $\begin{array}{l}\text { Nup58_mEGFP(- } \\
8)^{\star * *}\end{array}$ & Nup58 & 409 & 5 & -8 & RKMEELFT & $\begin{array}{l}\text { Fig. 2C; } \\
\text { Fig. 3C }\end{array}$ \\
\hline $\begin{array}{l}\text { Nup54- } \\
\text { mEGFP }{ }^{494}(0)^{* * * *}\end{array}$ & Nup54 & $\begin{array}{l}\text { 494AA + } \\
\text { 5AA rigid }\end{array}$ & 5 & 0 & DIKLVAEAAAEEELFT & $\begin{array}{l}\text { Fig. 1F; } \\
\text { Fig. 3D }\end{array}$ \\
\hline Nup54-mEGFP494(1) & Nup54 & $\begin{array}{l}\text { 494AA + } \\
\text { 6AA rigid }\end{array}$ & 5 & 1 & $\begin{array}{l}\text { DIKLVAEAAAKEELF } \\
\underline{\text { I }}\end{array}$ & $\begin{array}{l}\text { Fig. 1F; } \\
\text { Fig. } \\
\text { S3G }\end{array}$ \\
\hline Nup54-mEGFP ${ }^{494}(2)$ & Nup54 & $\begin{array}{l}\text { 494AA + } \\
\text { 7AA rigid }\end{array}$ & 5 & 2 & $\begin{array}{l}\text { DIKLVAEAAAKEEEL } \\
\text { FT }\end{array}$ & $\begin{array}{l}\text { Fig. 1F; } \\
\text { Fig. S3H }\end{array}$ \\
\hline $\begin{array}{l}\text { Nup54-mEGFP494 } \\
\text { (flex0) }\end{array}$ & Nup54 & $\begin{array}{l}\text { 494AA + } \\
\text { 5AA flex }\end{array}$ & 5 & 0 & $\begin{array}{l}\text { DIKLVGGGGSEEELF } \\
\text { I }\end{array}$ & Fig. 1G \\
\hline $\begin{array}{l}\text { Nup54-mEGFP494 } \\
\text { (flex1) }\end{array}$ & Nup54 & $\begin{array}{l}\text { 494AA + } \\
\text { 6AA flex } \\
\end{array}$ & 5 & 1 & $\begin{array}{l}\text { DIKLVGGGGSSEEEL } \\
\text { FT }\end{array}$ & Fig. 1G \\
\hline $\begin{array}{l}\text { Nup54-mEGFP494 } \\
\text { (flex2) }\end{array}$ & Nup54 & $\begin{array}{l}\text { 494AA + } \\
\text { 7AA flex }\end{array}$ & 5 & 2 & $\begin{array}{l}\text { DIKLVGGGGSSGEE } \\
\text { LFT }\end{array}$ & Fig. 1G \\
\hline $\begin{array}{l}\text { Nup54_mEGFP }{ }^{494}(- \\
\text { 4) }\end{array}$ & Nup54 & 494 & 4 & -4 & DIKLVGEELFT & Fig. 2D \\
\hline
\end{tabular}




\begin{tabular}{|l|l|l|l|l|l|l|}
\hline $\begin{array}{l}\text { Nup54_mEGFP } \\
\text { 5) }\end{array}$ & Nup54 & 494 & 5 & -5 & DIKLVEELFT & Fig. 2D \\
\hline $\begin{array}{l}\text { Nup54_mEGFP494(- } \\
6)\end{array}$ & Nup54 & 494 & 6 & -6 & DIKLVELFT & Fig. 2D \\
\hline $\begin{array}{l}\text { Nup54_mEGFP510(- } \\
4)^{* * * * *}\end{array}$ & Nup54 & 510 & 4 & -4 & GGVFSGEELFT & $\begin{array}{l}\text { Fig. 2E; } \\
\text { Fig. 3E }\end{array}$ \\
\hline $\begin{array}{l}\text { Nup54_mEGFP510(- } \\
5)\end{array}$ & Nup54 & 510 & 5 & -5 & GGVFSEEEFT & Fig. 2E \\
\hline $\begin{array}{l}\text { Nup54_mEGFP510(- } \\
6)\end{array}$ & Nup54 & 510 & 6 & -6 & GGVFSELFT & Fig. 2E \\
\hline
\end{tabular}

* This construct is the Nup133_mEGFP construct used in the transient transfection starvation experiment

3 in Figure 3.

$4 \quad * *$ This construct is the Nup93_mEGFP construct used in the transient transfection starvation experiment

5 in Figure 3.

$6{ }^{* * *}$ This construct is the Nup58_mEGFP construct used in the transient transfection starvation experiment

7 in Figure 3. **** This construct is the Nup54_mEGFP494 construct used in the transient transfection starvation

9 experiment in Figure 3.

$10^{* * * *}$ This construct is the Nup54_mEGFP510 construct used in the transient transfection starvation 11 experiment in Figure 3. 
Table S2: Crispr Nup-mEGFP Cell Lines

\begin{tabular}{|c|c|c|c|c|c|c|}
\hline Construct Name & Nup & $\begin{array}{l}\text { Position of } \\
\text { mEGFP } \\
\text { within Nup }\end{array}$ & $\begin{array}{l}\text { Size of } \\
\text { mEGFP } \\
\text { Deletio } \\
n \\
\text { (AAs) }\end{array}$ & $\begin{array}{l}\text { Net } \\
\text { Linker } \\
\text { Size } \\
\text { (AAs) }\end{array}$ & $\begin{array}{l}\text { Amino Acid Linker } \\
\text { Sequence } \\
\text { Nup Sequence in } \\
\text { Blue } \\
\text { mEGFP Sequence in } \\
\text { Green }\end{array}$ & Figures \\
\hline $\begin{array}{l}\text { Nup133_mEGFP(- } \\
9)^{\dagger}\end{array}$ & $\begin{array}{l}\text { Nup13 } \\
3\end{array}$ & $\begin{array}{l}\text { Carboxyl- } \\
\text { Terminus }-3\end{array}$ & 6 & -9 & EYYVQELFT & $\begin{array}{l}\text { Fig. } \\
4 \mathrm{C}, \mathrm{E}, \mathrm{G}, \mathrm{I} \\
\text {,K; Fig. } \\
5\end{array}$ \\
\hline Nup133_mEGFP(-8) & $\begin{array}{l}\text { Nup13 } \\
3\end{array}$ & $\begin{array}{l}\text { Carboxyl- } \\
\text { Terminus -2 }\end{array}$ & 6 & -8 & EYYVQGELFT & $\begin{array}{l}\text { Fig. 4C; } \\
\text { Fig. S5A }\end{array}$ \\
\hline $\begin{array}{l}\text { Nup54- } \\
\text { mEGFP494}(0)^{\dagger \dagger}\end{array}$ & Nup54 & $\begin{array}{l}\text { 494AA + } \\
\text { 5AA rigid }\end{array}$ & 5 & 0 & DIKLVAEAAAEELFT & $\begin{array}{l}\text { Fig. } \\
\text { 4D,F,H, } \\
\text { J,L; Fig. } \\
5\end{array}$ \\
\hline Nup54-mEGFP $494(1)$ & Nup54 & $\begin{array}{l}\text { 494AA + } \\
\text { 6AA rigid }\end{array}$ & 5 & 1 & DIKLVAEAAAKEELFF & $\begin{array}{l}\text { Fig. 4D; } \\
\text { Fiq. S5B }\end{array}$ \\
\hline Nup54-mEGFP ${ }^{494}(2)$ & Nup54 & $\begin{array}{l}\text { 494AA + } \\
\text { 7AA rigid }\end{array}$ & 5 & 2 & $\begin{array}{l}\text { DIKLVAEAAAKEEELF } \\
\text { T }\end{array}$ & $\begin{array}{l}\text { Fig. 4D; } \\
\text { Fig. S5C }\end{array}$ \\
\hline
\end{tabular}

$3+$ This cell line is the Nup133_mEGFP cell line used for the experiments to reduce transport in Figure 4

4 and the experiments to manipulate transport factors in Figure 5.

$5 \quad$ t† This cell line is the Nup54_mEGFP cell line used for experiments to reduce transport in Figure 4 and

6 the experiments to manipulate transport factors in Figure 5. 